\title{
In Vivo Detoxification of Ochratoxin A by Highly Porous Magnetic Nanocomposites Prepared from Coconut Shell
}

\section{-Author(s)}

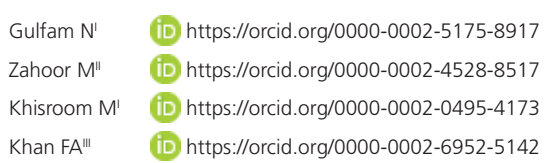

Department of Zoology, Peshawar Uni-versity, Khyber Pakhtunkhwa, Pakistan.

Department of Chemistry, University of Malakand, Chakdara, Dir Lower, 18800 KPK, Pakistan.

III Principal Abbotabad International College of Pharmacy AIMI, Abbotabad, KPK, Pakistan.

\section{-Mail Address}

Corresponding author e-mail address Dr Muhammad Zahoor

Department of Chemistry, University of Malakand Chakdara Dir Lower KPK,

Pakistan.

Phone: (86)-0371-67756513

Email: mohammadzahoorus@yahoo.com

\section{aKeywords}

Serum alanine transferase, alkaline phosphatase, Clinical signs, Gross lesions.

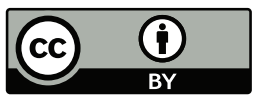

Submitted: 03/December/2017 Approved: 23/April/2018

\section{ABSTRACT}

The current study was conducted to elucidate the clinical effect of ochratoxin A in Gallus gallusdomesticus and to ameliorate its toxic effects by the development and characterization of highly porous carbon-based adsorbent derived from coconut shell. A series of experiments were performed on one day-old chicks (Group A to F). Clinical signs of the positive control (group B fed on 400 ppb ochratoxin A contaminated feed) included depression, diarrhea, increased water intake, low body weight, high degree of genotoxicity, swollen and hemorrhagic kidneys and liver.

Serum alanine amino transferase (ALT), alkaline phosphatase (ALP), creatinine and urea concentration significantly increased while albumin, globulin and total proteins were found significantly low. All these lead to increased mortality.

Among adsorbents treated groups, chickens in group $C(0.3 \%$ of the prepared adsorbent mixed in OTA contaminated feed) and D $(0.6 \%$ of the prepared adsorbent mixed in Ochratoxin A (OTA) contaminated feed) showed very low pathological effects while group $E(0.9 \%$ of the prepared adsorbent mixed in OTA contaminated feed) and $F(1.2 \%$ of the prepared adsorbent mixed in OTA contaminated feed) showed normal behavior, blood biochemistry and well maintained histological structure like that in group A. As a whole, the adsorbents treated groups fed with ochratoxin A contaminated feed, supplemented with different levels of the prepared adsorbent helped to ameliorate the toxic effects of OTA and nearly showed normal clinical signs.

\section{INTRODUCTION}

Ochratoxins are a group of mycotoxins produced as secondary metabolites by toxigenic mycobiota. Structurally, all the ochratoxins consist of derivatives of polyketide dihydrocoumarin moiety linked through the 7- carbon group to L- $\beta$-phenylalanine by an amide bond (Vander et al., 2001). Avian ochratoxicosis is a disease that affects birds, which the causative agent is ochratoxins. Sensitivity to ochratoxin A (OTA) may vary among different species of birds. Vulnerability to OTA induced diseases may also vary depending on cell type and sex of a species, for instance renal tissue damage caused by ochratoxin $A$ is severe when_compared to the rest of tissue damage. OTA contamination in poultry feed results in growth retardation, low efficiency and feed intake (Peckham et al., 1971) and high rate of mortality (Elaroussi et al., 2006). OTA is also nephrotoxic and causes gross lesions and severe renal hemorrhages as well as hepatica tissue hemorrhage that finally leads to impaired renal function. Besides nephropathic effects, ochratoxin A have negative effects on the liver. Several authors have reported hepatotoxicity, cytoplasmic vacuolation of hepatocytes and fatty deposition in the liver of poultry chicks when fed with ochratoxin A contaminated feed (Elaroussi et al., 2002). 
Over the former few decades, literature has been published to reveal the approaches involved in ochratoxin A carcinogenicity and toxicity (Schilteret al., 2005). There has been significant discussion about the genotoxic and carcinogenic potential of OTA (KuiperGoodman \& Scott 1989). Numerous researchers and proficient groups have determined that OTA is genotoxic as well as nephrotoxic (Pfohl-Leszkowicz \& Manderville 2012). Control and prevention of ochratoxin contamination is necessary. If it still occurs, then it is compulsory to detoxify it prior to usage. various chemical, physical and biological measures and treatments have been taken into account for ochratoxin detoxification.

One of the effective methods to treat mycotoxicosis in animals and poultry birds is the application of adsorbents (Medinaet al., 2007). Over the last few decades, there has been a constant growing attention and exploration of efforts in the manufacturing, functionalization, characterization, molecular composition and design of extremely porous carbonbased adsorbents plus their application against mycotoxin (Arenillas et al., 2005). Although activated carbon has high surface area and can effectively adsorb mycotoxins in the gastrointestinal tract of chicks but also causes dehydration and minerals depletion. Also, it cannot easily be collected from feces after treatment and will cause secondary environmental problems as the adsorbed mycotoxin will reach the human body through food chain. Magnetic activated carbon on the other hand has comparatively less surface area when compared to activated carbon and do not cause dehydration and mineral deficiency in chicks. Also due to magnetic character it can be easily collected from feces through magnetic processes (Khan \& Zahoor 2014).

Keeping in view the importance of ochratxin $A$ in poultry industry, the present study was designed to determine the in vivo efficacy of the novel adsorbent prepared from coconut shell in broiler chicks.

\section{MATERIALS AND METHODS}

\section{Preparation and characterization of adsor- bent}

In this study a novel magnetic adsorbent from coconut shell was prepared by following the methods of Khan et al. (2014) with slight modification. Coconut shell was utilized as bulk material for the preparation of carbon-based adsorbents. The material was crushed and soaked in ethanolic $\mathrm{FeCl}_{3} \cdot 6 \mathrm{H}_{2} \mathrm{O}(10 \% \mathrm{w} / \mathrm{v})$. When half of an hour has passed, the biomass is_taken out and dried at room temperature for about 24 hours. The dried materials were then passed through pyrol fumigation for 48 hours for pyrolysis. The pyrol treated biomass was then shifted to an oven for drying at 100 ${ }^{\circ} \mathrm{C}$ for 5 hours. After that material was taken out from the oven and shifted to a specially designed chamber consisting of a stainless steel container comprising of electric heater, a wire gauze, inlet, exhaust outlet and supply of nitrogen gas (to provide inert environment) and was charred. Temperature in the chamber was $500{ }^{\circ} \mathrm{C}$, which was set in such a manner that there was $10{ }^{\circ} \mathrm{C}$ increase in temperature/min. The material was kept in the chamber for 2 hours to accomplish the process. When the processed sample was subjected to magnetic bar all the sample contents were attracted toward it. This clearly indicated that formation of Iron oxide has occurred. After the preparation the adsorbent was treated with different concentrations of $\mathrm{HNO}_{3}$ solution to remove $50 \%$ of the iron contents. The highly porous adsorbent prepared was then characterized by different instrumental techniques (surface area analyzer, FTIR (Fourier-transform infrared spectroscopy), SEM (Scanning electron microscope), XRD (X-ray diffraction), TG/DTA (Thermogravimetric/ differential thermal analysis), EDX (Energy-dispersive X-ray spectroscopy) etc.).

\section{Experimental protocol for induction of ochratoxin A in broiler chicks}

A total of 600 , one day old broiler chicks (Gallus gallusdomesticus) were purchased from a_local hatchery (Rawalpendi, Pakistan) and were reared in well managed and hygienic conditions. All these chicks were divided in six equal groups (A-F) containing100 chicks in each and access to fresh water was given ad libitum.

Chicks in group A were provided with ochratoxin A free diet during the whole period of the experiment and served as (negative control). Chicks in group B were provided with ochratoxin A $(400 \mu \mathrm{g} / \mathrm{kg})$ contaminated feed during the whole period of the experiment and served as (positive control). Ochratoxin A was produced on maize by following the protocols of Shotwell et al. (1966). The details of the method were as follow:

One liter round bottom flask was taken and $50 \mathrm{~g}$ of crushed maize along with $25 \mathrm{ml}$ of distilled water was added to it. The flask (containing maize and water) was shaken well and then placed at room temperature for 2 hours. About $3 \mathrm{ml}$ of spores were taken from PDA slant and were inoculated in the flask and then 


\section{In Vivo Detoxification of Ochratoxin A by Highly Porous Magnetic Nanocomposites Prepared from Coconut Shell}

On the other hand, blood serum biochemistry was analyzed by slaughtering three individual chicks from each group at the end of the $1^{\text {st }}$ to the $6^{\text {th }}$ week of the experiment.

Data was statistically analyzed for mean, standard error of mean, standard deviation, descriptive statistics, all pairwise comparison tests and completely randomized tests by using software STATISTIX 8.1.

\section{Single cell gel electrophoresis (SCGE) I Comet Assay}

Genotoxic effect of OTA was determined by following the protocols of Singh et al. (1988) with slight modifications. The blood was collected in a $5 \mathrm{ml}$ sterilized syringes from the_brachial vain of the selected chicks. For genotoxicity of kidney and liver, about $1 \mathrm{~g}$ of each tissue was grinded separately through grinder machine and about 5-6 $\mathrm{ml}$ of phosphate buffer saline (PBS) was added to the grinded tissues to obtain the solution.

To the single coated slide, $75 \mu \mathrm{L}$ of low melting point agarose (LMPA, $1.5 \%$ ) mixed with $15 \mu \mathrm{L}$ of blood was added. A cover slip was placed on it and the slide was put on an ice pack for $\sim 5$ to 10 minutes. The cover slip was removed and a third LMPA layer of $85 \mu \mathrm{L}$ was added to the same slide. After 10 min on the ice pack, the coverslip was removed and slides were shifted to freshly made Lysing solution poured in a glass tray overnight at $4{ }^{\circ} \mathrm{C}$. After electrophoresis the slides were rinsed with a Neutralization Buffer and stained with $70 \mu \mathrm{L}$ DNA specific fluorescent dye Acridine Orange (20 $\mu \mathrm{g} / \mathrm{ml})$. Before observing the slides, the excess of water on the edges and back of the slides was blotted away. From every slide 100 cells were randomly selected and images (Figure 1) were taken at $400 x$ by using fluorescent microscope (Nikon Eclipse 80 i) equipped

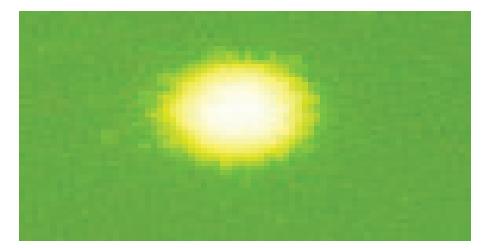

Class 0

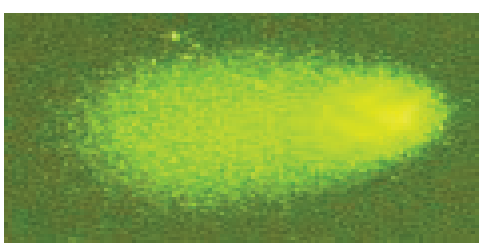

Class 3

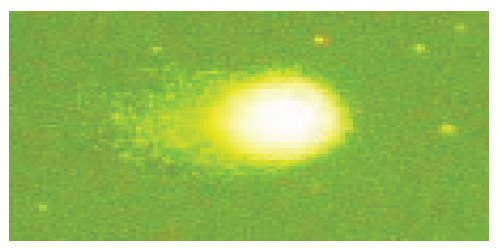

Class 1

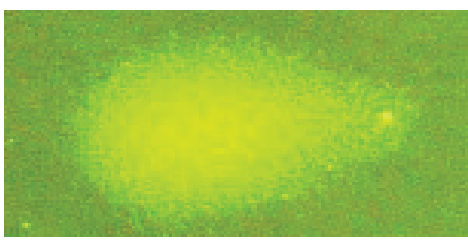

Class 4

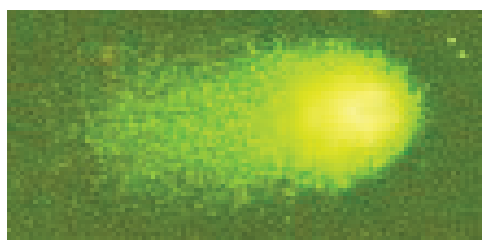

Class 2

Figure 1 - Different comet classes (0-4) and level of DNA damage. 
Gulfam N, Zahoor M, Khisroom M, Khan FA
In Vivo Detoxification of Ochratoxin A by Highly Porous Magnetic Nanocomposites Prepared from Coconut Shell with an excitation filter of 450-490 nm. Comet tail lengths (consisting of nuclear region and tail) were scored visually into 5 Comet Classes:

Comet Class 0 (no damage, hence no tail), Comet Class 1 (tail up to 1.5 times the diameter of the comet nucleus), Class 2 (tail 1.5-2.0 times the diameter of the comet nucleus), Class 3 (tail 2.0-2.5 times the diameter of the comet nucleus) and Class 4 (maximally damaged with total DNA in its tail).

A final overall total comet score for all 100 cells was obtained by summing up the number of cells in each class times the class number, giving a rating between 0-4 i.e.

TCS $=0(n)+1(n)+2(n)+3(n)+4(n)$, Where $(n)$ indicate the number of cells in each class.

\section{Tissue processing for histological evalua- tion}

For histological evaluation the tissues were processed manually according to the procedures of laboratory methods in histotechnology (Prophet \& Edna 1992).

After dissecting the selected broiler chick, kidney and liver tissue samples were immediately transferred to $10 \%$ neutrally buffered formalin and remained in fixative for a minimum of 48 hours. Then these tissues were dehydrated in different concentrations of ethanol solution and finally transferred to a clearing solution. The dehydrated and cleared tissues were infiltrated with melted paraffin and embedded in stainless steel (S.S) moulds. The moulds were then placed in a freezer and when completely solidified, the tissue blocks were removed. The tissues were sectioned through a rotary microtome and stained with Harris hematoxylin and eosin-phloxine solution. Photomicrographs of tissues sections were taken on a digital camera attached to the microscope.

\section{Biochemistry of blood}

Serum biochemical parameters such as alanine transferase (ALT), serum alkaline phosphatase (ALT), Serum albumen, total proteins, globulin, serum urea and creatinine were determined by using commercially available kits of RANDOX.

\section{Clinical signs}

Clinical signs and behavior of poultry chicks (attentiveness, attraction to water and feed, and consistency of feces) were analyzed by score from 0 to 3 based upon (0) absences, (1) minor, (2) average and
(3) extreme. These interpretations and observations were kept in record at end of each week of experiment.

\section{RESULTS AND DISCUSSION}

\section{Highly porous carbon nanocomposite}

The prepared magnetic adsorbent has a high Langmuir surface area of $989.12 \mathrm{~m}^{2} / \mathrm{g}$ with $50 \%$ magnetic character as well. The adsorbent was characterized by different instrumental techniques (Figure 2) and used in in-vitro for the detoxification of ochratoxin A (our unpublished paper). SEM picture confirmed that the excess of deposited Iron oxide was removed when treated with $\mathrm{HNO}_{3}$ thus leaving the nano-pores in the prepared sample. The pores size was noted below $100 \mathrm{~nm}$ however the remaining of iron oxide particles after treatment with $\mathrm{HNO}_{3}$ solution as white patches can also be seen in the highly porous carbon nano-composite prepared from coconut shell. The surface morphology of Iron oxide nanoparticles was carried out by Sneha et al. (2015) and confirmed the porous nature of the product through SEM.

The XRD patterns have indicated that iron oxide (magnetite) is deposited on the activated carbon, while EDX showed the peaks of Fe and O. The EDX pattern in figure 2 shows Fe 0.7, 2.6, 6.5 and $7 \mathrm{KeV}$ which confirms the presence of iron in the composite structure. In our previous work similar EDX spectra of the composite was obtained (Zahoor \& Khan, 2014). The TG/DTA pattern in figure 2 shows a gradual loss of weight up to $390{ }^{\circ} \mathrm{C}$. An abrupt fall in loss of weight was noted from $400{ }^{\circ} \mathrm{C}$ to $595^{\circ} \mathrm{C}$, whereas no loss of weight was observed above $600^{\circ} \mathrm{C}$.

The mid and far IR spectra of highly porous carbon nano-composite, prepared from coconut shell, are presented in figure 2 . The FTIR spectrum exhibit band at $3412 \mathrm{~cm}^{-1}$ showing stretching vibration of $\mathrm{O}-\mathrm{H}$ whereas $2600 \mathrm{~cm}^{-1}$ representing the aliphatic $\mathrm{C}-\mathrm{H}$. The bands between 1200 to $1000 \mathrm{~cm}^{-1}$ can be attributed to $C=O$ and $C=C$ respectively (Wang et al., 2009). Other significant bands at 1002.98 and 1080 and $1190 \mathrm{~cm}^{-1}$ are ascribed to C-O stretching as reported in earlier studies (Hadi et al., 2015). The band at 587 $\mathrm{cm}^{-1}$ corresponds to stretching of $\mathrm{Fe}-\mathrm{O}$ deposited in the pores and surface of the prepared adsorbent from coconut shell as reported previously (Sneha et al., 2015, Zahoor \& Khan, 2015).

The novel adsorbent was then used for the in-vivo detoxification of ochratoxin A. The in-vivo results are described below. 
Gulfam N, Zahoor M,

Khisroom M, Khan FA

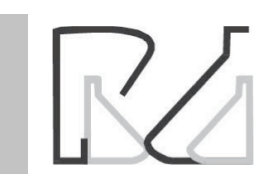

In Vivo Detoxification of Ochratoxin A by Highly Porous Magnetic Nanocomposites Prepared from Coconut Shell

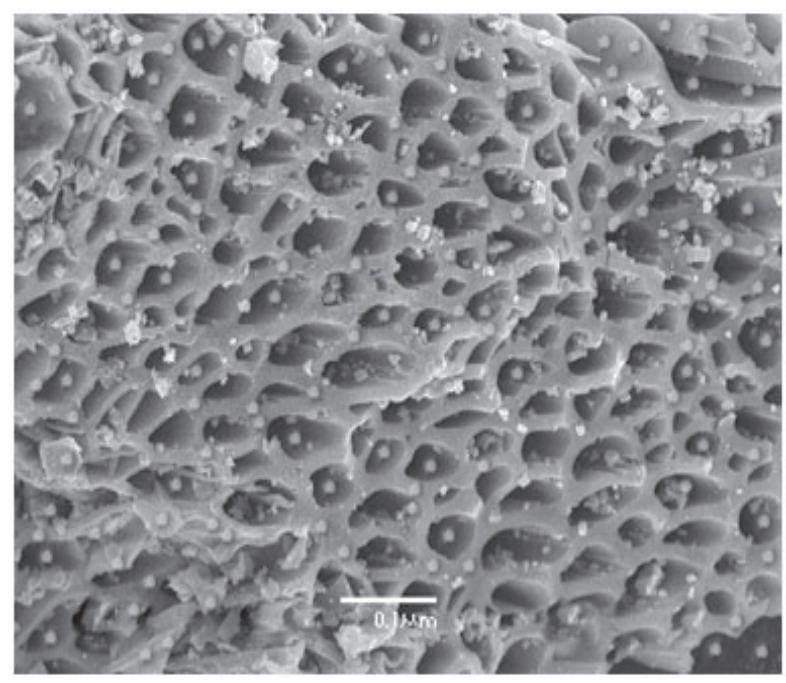

ESM

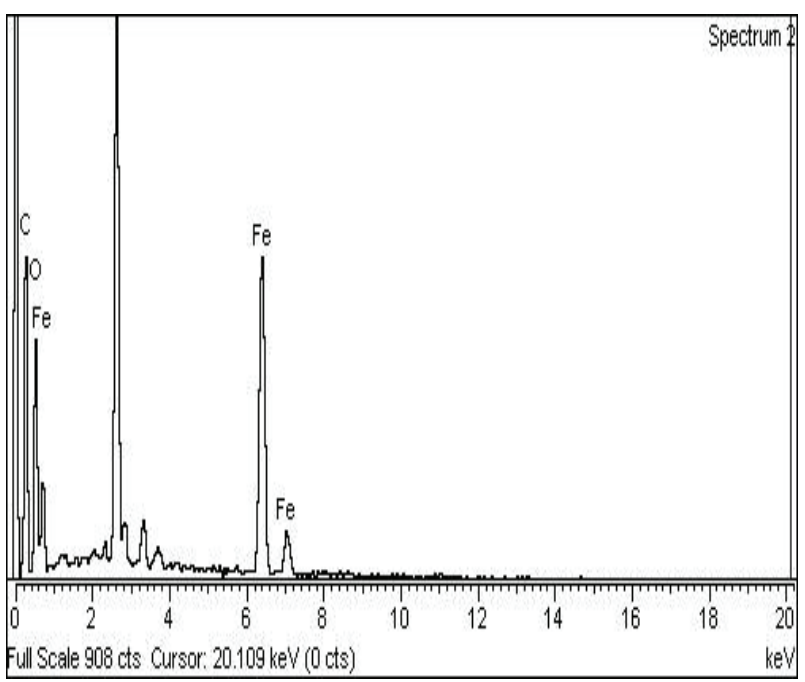

EDX

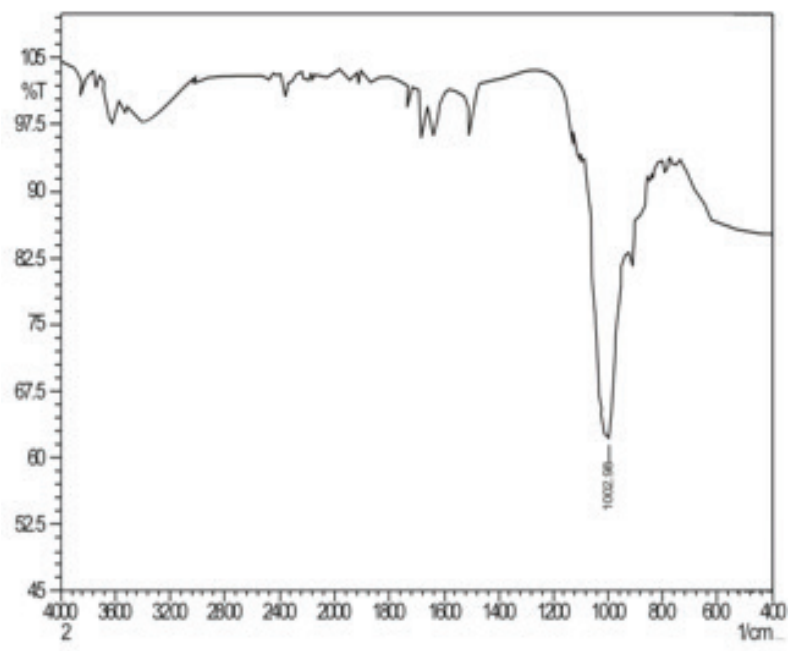

Mid FTIR spectra

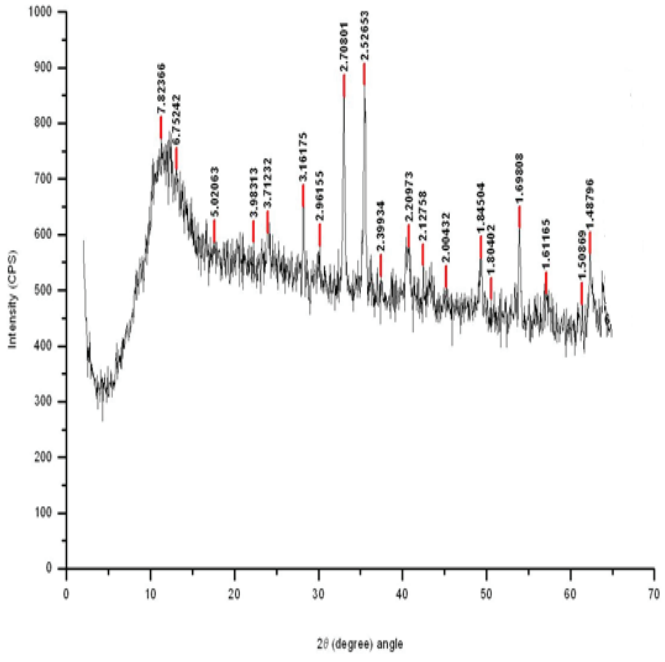

XRD

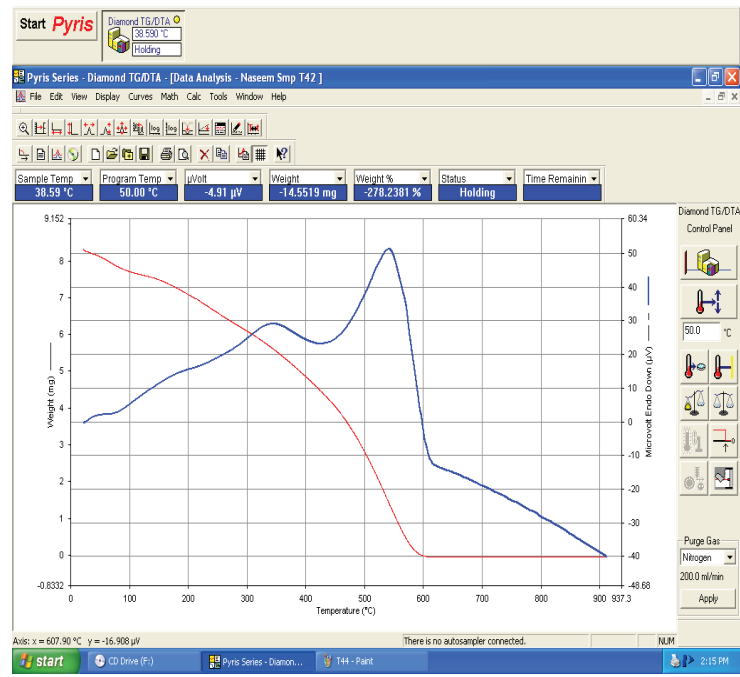

TG/DTA

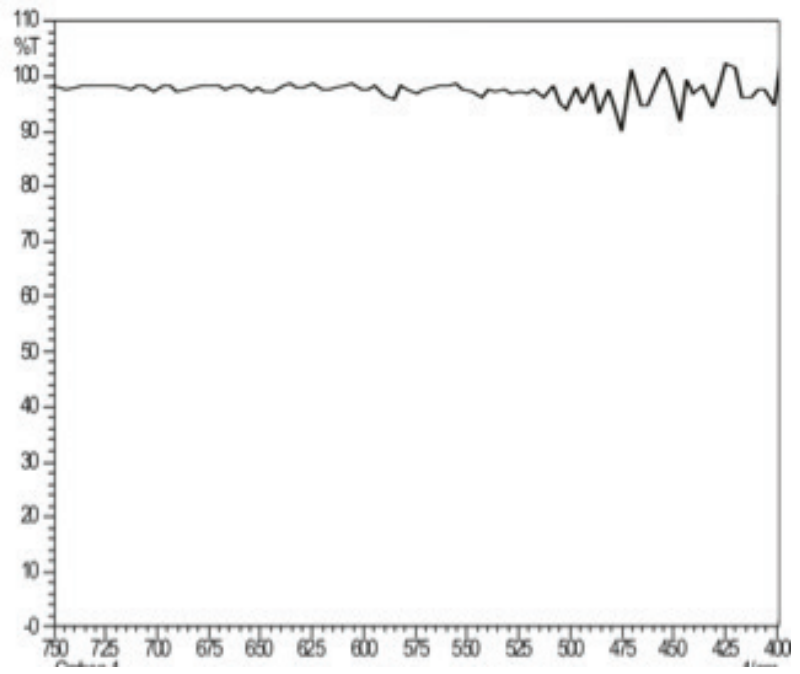

Far FTIR spectra

Figure 2 - Photomicrographs showing characterization of highly porous Iron oxide carbon nano-composite prepared from coconut shell. 


\section{Ochratoxin A induced genotoxicity and its detoxification in broiler chicks}

Blood, kidney and liver tissue samples of broiler chicks from each group were taken and treated to estimate DNA damage caused by ochratoxin (1st week to the $6^{\text {th }}$ week). The DNA damage observed in the blood, kidney and liver tissue samples in group B and C was significantly greater than that estimated in group A, E and $F$ (Table 1s to $6 \mathrm{~s}$ ). The blood, kidney and liver samples in group B and $C$ showed higher mean frequency of total comet score (TCS) and comet class per 100 cells as compared with those from group A, E and $\mathrm{F}$. Besides value of comet class 0 was highest in the blood in group A (negative control) while lowest mean values of comet class 0 were observed in the blood in group $B$ birds (positive control). During the whole experimental period blood samples of broilers in group A (negative control) showed maximum of class (0) and minimum score of class (4). Thus, maximum mean value of class (0) is shown by group A. Mean value of comet class (4) in blood samples of broilers in group $B$ (positive control) was low during the first week but with the increase in exposure duration, there was an increase in score of class (4). The increase in score of class (4) is indication by high degree of genotoxicity. The value of TCS at the end of the $6^{\text {th }}$ week was $221.97 \pm 9.9$. Thus, the maximum total comet score (TCS) and high degree of genotoxicity was shown by the chickens in group $B$ followed by group $C$. The value of TCS of chickens in group A, E and F was 24.94 \pm 4 , $43.06 \pm 7.6$ and $26.96 \pm 11.2$ respectively at the end of the last week of the experiment which represents least TCS. The sequence for total comet score (TCS) in the blood sample of different groups at the end of the experiment was in the following order $B>$ group $C>$ group $D>$ group $E>$ group $F>$ group $A$. Thus, the higher total comet score is shown by group $B$.

Likewise, liver samples of broilers in group $A$ showed least TCS value of $26.96 \pm 4$.9. Group $B, C$ and D showed TCS of $225.99 \pm 6.1,81.3 \pm 11.6$ and $65.3 \pm 17.3$ respectively, however group $\mathrm{E}$ and $\mathrm{F}$ represented TCS of $43.97 \pm 6.4$ and $27.61 \pm 5.2$ respectively. Thus, high degree of DNA damage is observed in the liver sample in group $B$ and $C$. The sequence for total comet score (TCS) in the liver sample of different groups at the end of the experiment was in the following_order $B>$ group $C>$ group $D>$ group $E>$ group $F>$ group $A$. Thus, the higher total comet score is shown by group $B$.

Furthermore, kidney samples of broilers in group $A$ showed maximum score of class (0) minimum score of class (4) with TCS of $28.32 \pm 6.1$. The value of total comet score (TCS) in group B was $235.64 \pm 7.9$ while that in group $C$ and D was $82.29 \pm 5.6$ and $66.63 \pm 8.6$ respectively. Likewise, kidney of chicks from group $\mathrm{E}$ and F presented low TCS of $43.98 \pm 3.4$ and $31.99 \pm 4.7$ respectively. The sequence for total comet score (TCS)

Table 1s - 1st week

Group

Ochratoxin A 400 $\mu \mathrm{g} / \mathrm{kg}$ feed

\begin{tabular}{|c|c|c|c|c|c|c|c|}
\hline \multirow{7}{*}{ Blood } & Comet class and TCS & $A$ & $B$ & $C$ & $\mathrm{D}$ & $E$ & $\mathrm{~F}$ \\
\hline & Class 0 & $92.33 \pm 2.8$ & $59.33 \pm 1.6$ & $71 \pm 2.4$ & $71.66 \pm 2.1$ & $75.66 \pm 2.2$ & $91.33 \pm 1.6$ \\
\hline & Class 1 & $3.33 \pm 1.6$ & $21.66 \pm 1.4$ & $18 \pm 2.4$ & $18.66 \pm 1.7$ & $19 \pm 1.2$ & $2.66 \pm 2.0$ \\
\hline & Class 2 & $3.66 \pm 2.6$ & $8.33 \pm 0.8$ & $7.33 \pm 0.4$ & $5 \pm 0.7$ & $2.33 \pm 0.4$ & $3.66 \pm 1.4$ \\
\hline & Class 3 & $0.33 \pm 0.4$ & $7.66 \pm 0.8$ & $1.33 \pm 0.4$ & $1.66 \pm 0.8$ & $2.33 \pm 1.6$ & $0.66 \pm 0.4$ \\
\hline & Class 4 & $0.33 \pm 0.4$ & $3 \pm 0.7$ & $2.33 \pm 0.8$ & $1.33 \pm 0.4$ & $0.66 \pm 0.4$ & $1.66 \pm 0.4$ \\
\hline & TCS & $12.98 \pm 9.6$ & $73.3 \pm 8.2$ & $45.9 \pm 7.6$ & $38.7 \pm 7.1$ & $33.3 \pm 0.8$ & $18.64 \pm 7.6$ \\
\hline \multirow{6}{*}{ Liver } & Class 0 & $91.33 \pm 1.0$ & $58.66 \pm 1.0$ & $69.66 \pm 0.4$ & $67.66 \pm 4.7$ & $73.66 \pm 3.2$ & $90.66 \pm 2.2$ \\
\hline & Class 1 & $4.33 \pm 0.4$ & $21.66 \pm 1.0$ & $18.33 \pm 2.8$ & $20.66 \pm 1.6$ & $20.33 \pm 3.5$ & $2.66 \pm 1.0$ \\
\hline & Class 2 & $3.33 \pm 1.0$ & $8.66 \pm 0.4$ & $8 \pm 2.4$ & $4.66 \pm 1.0$ & $4.66 \pm 1.6$ & $2.33 \pm 1.0$ \\
\hline & Class 3 & $0.66 \pm 0.4$ & $7.66 \pm 0.4$ & $1.66 \pm 0.4$ & $1.66 \pm 0.8$ & $0.66 \pm 0.4$ & $2.66 \pm 0.8$ \\
\hline & Class 4 & $0.33 \pm 0.40$ & $3.33 \pm 0.4$ & $2.33 \pm 0.4$ & $1 \pm 0$ & $0.66 \pm 0.4$ & $1 \pm 0.7$ \\
\hline & TCS & $14.43 \pm 7$ & $75.2 \pm 4.6$ & $48.6 \pm 10.4$ & $38.9 \pm 6$ & $34.2 \pm 9.5$ & $19.32 \pm 8.2$ \\
\hline \multirow{6}{*}{ Kidny } & Class 0 & $92.33 \pm 1.4$ & $58.66 \pm 1.0$ & $63.33 \pm 1.0$ & $67.33 \pm 0.8$ & $74.33 \pm 1.0$ & $91 \pm 1.8$ \\
\hline & Class 1 & $2.33 \pm 0.4$ & $21.66 \pm 0.8$ & $24.3 \pm 0.4$ & $24.66 \pm 1.6$ & $18.33 \pm 0.4$ & $2.33 \pm 0.4$ \\
\hline & Class 2 & $4.33 \pm 1.7$ & $8 \pm 0.7$ & $9 \pm 2.1$ & $4.66 \pm 1.0$ & $4.66 \pm 0.8$ & $4 \pm 2.5$ \\
\hline & Class 3 & $0.33 \pm 0.4$ & $8.33 \pm 0.4$ & $1.66 \pm 0.4$ & $1.66 \pm 0.4$ & $1.66 \pm 0.8$ & $1.66 \pm 0.8$ \\
\hline & Class 4 & $0.66 \pm 0.4$ & $3.33 \pm 0.4$ & $2.33 \pm 0.4$ & $1.66 \pm 0.8$ & $1 \pm 0$ & $1 \pm 0.7$ \\
\hline & TCS & $14.65 \pm 6.6$ & $75.9 \pm 5$ & $56.6 \pm 7.4$ & $45.6 \pm 8$ & $36.6 \pm 7.6$ & $19.2 \pm 10.6$ \\
\hline
\end{tabular}

$\mathrm{A}=$ pure feed, $\mathrm{B}=$ OTA contaminated feed $(400 \mu \mathrm{g}$ of OTA $/ \mathrm{kg}$ of feed), $\mathrm{C}=$ OTA contaminated feed $(400 \mu \mathrm{g}$ of OTA/kg of feed $)+0.3 \%$ Adsorbent, $\mathrm{D}=\mathrm{OTA}$ contaminated feed $(400 \mu \mathrm{g}$ of OTA $/ \mathrm{kg}$ of feed $)+0.6 \%$ Adsorbent, $E=$ OTA contaminated feed $(400 \mu \mathrm{g}$ of OTA $/ \mathrm{kg}$ of feed $)+0.9 \%$ Adsorbent, F $=0$ OTA contaminated feed (400 $\mu \mathrm{g}$ of OTA $/ \mathrm{kg}$ of feed) $+1.2 \%$ Adsorbent. 
in the kidney samples of different groups at the end of the experiment was in order the following order $B$ $>$ group $C>$ group $D>$ group $E>$ group $F>$ group $A$. Thus, the higher total comet score is shown by group B. While the overall total comet classes scored in blood, liver and kidney of broilers (table 1s to 6s) studied was in order the following order: kidney > liver > blood.

Genotoxicity is a detrimental activity that brings about alterations in cell integrity by affecting its genome (Arbillaga et al., 2007). Several authors and expert groups have concluded that OTA is genotoxic (Sawale et al., 2009). In the present study, groups C, D, E and F were kept on ochratoxin A contaminated feed $(400 \mu \mathrm{g}$ OTA/Kg) supplemented with $0.3 \%, 0.6 \%, 0.9 \%$ and
$1.2 \%(\mathrm{w} / \mathrm{w})$ of the prepared adsorbents respectively. Therefore the rate of total comet score and genotoxicity in blood, kidney and liver samples in group $C$ and $D$ was comparatively less than group $B$, while group $F$ showed the least value of total comet score followed by group $\mathrm{E}$ (among all the adsorbent treated groups). Therefore, group $E$ and $F$ were safe from the genotoxic effects of ochratoxin A which could be correlated to the fact that maximum ochratoxin $A$ has been adsorbed by the adsorbent prepared from coconut shell through the alimentary canal of chicks, which later on passed along with feces. Thus, our findings show maximum efficacy of the prepared adsorbent in ameliorating the toxic effects of ochratoxin A in Gallus gallusdomesticus.

Table 2s - $2^{\text {nd }}$ week

\begin{tabular}{|c|c|c|c|c|c|c|c|}
\hline & \multicolumn{7}{|c|}{ Group } \\
\hline & \multicolumn{7}{|c|}{ Ochratoxin A 400 $\mathrm{g} / \mathrm{kg}$ feed } \\
\hline & Comet class and TCS & A & B & $C$ & $\mathrm{D}$ & $E$ & $\mathrm{~F}$ \\
\hline \multirow{6}{*}{ Blood } & Class 0 & $91.66 \pm 1.0$ & $49 \pm 0.7$ & $56.6 \pm 4.1$ & $69.33 \pm 0.4$ & $76.6 \pm 0.81$ & $89.6 \pm 1.0$ \\
\hline & Class 1 & $1.33 \pm 0.4$ & $16 \pm 1.2$ & $23.6 \pm 1.6$ & $21.33 \pm 0.8$ & $19.6 \pm 1.0$ & $2.66 \pm 0.4$ \\
\hline & Class 2 & $4 \pm 0.7$ & $12.6 \pm 0.4$ & $9.3 \pm 0.8$ & $3 \pm 0.7$ & $2.6 \pm 0.4$ & $2.33 \pm 0.4$ \\
\hline & Class 3 & $0.66 \pm 0.4$ & $10.3 \pm 1.0$ & $3 \pm 0.7$ & $3.33 \pm 0.4$ & $0.6 \pm 0.4$ & $3 \pm 0$ \\
\hline & Class 4 & $2.33 \pm 0.4$ & $12 \pm 0.7$ & $4 \pm 1.2$ & $3 \pm 0.7$ & $0.3 \pm 0.4$ & $2.33 \pm 0.4$ \\
\hline & TCS & $20.63 \pm 4.6$ & $129.3 \pm 7.8$ & $67.32 \pm 10.1$ & $28.28 \pm 4.6$ & $28.28 \pm 4.6$ & $25.6 \pm 2.8$ \\
\hline \multirow{6}{*}{ Liver } & Class 0 & $90.66 \pm 1.4$ & $49.3 \pm 0.4$ & $61.6 \pm 1.4$ & $68.66 \pm 1.0$ & $76 \pm 1.2$ & $88.66 \pm 0.4$ \\
\hline & Class 1 & $3.33 \pm 0.4$ & $15 \pm 0.7$ & $22.3 \pm 1.6$ & $21.33 \pm 1.0$ & $19.33 \pm 1.6$ & $3 \pm 0.7$ \\
\hline & Class 2 & $3 \pm 0.7$ & $11.33 \pm 0.8$ & $8.3 \pm 0.4$ & $3.33 \pm 0.8$ & $2.66 \pm 0.4$ & $2.66 \pm 0.4$ \\
\hline & Class 3 & $0.66 \pm 0.8$ & $12 \pm 0.7$ & $3.6 \pm 0.8$ & $3.66 \pm 1.0$ & $1.66 \pm 0.4$ & $3 \pm 0$ \\
\hline & Class 4 & $2.33 \pm 0.4$ & $12.33 \pm 0.4$ & $4 \pm 0.7$ & $3 \pm 0.7$ & $0.33 \pm 0.4$ & $2.66 \pm 0.4$ \\
\hline & TCS & $20.63 \pm 5.8$ & $122.98 \pm 6$ & $65.97 \pm 7.6$ & $30.95 \pm 5.2$ & $30.95 \pm 5.2$ & $27.7 \pm 3.1$ \\
\hline \multirow{6}{*}{ Kidny } & Class 0 & $89 \pm 1.4$ & $55 \pm 2.5$ & $60.3 \pm 1.0$ & $64 \pm 1.2$ & $73.33 \pm 1.6$ & $88 \pm 1.2$ \\
\hline & Class 1 & $4.66 \pm 0.4$ & $17.33 \pm 1.0$ & $22 \pm 0.7$ & $25 \pm 1.4$ & $21.66 \pm 1.6$ & $3.33 \pm 0.4$ \\
\hline & Class 2 & $2.66 \pm 0.4$ & $11.33 \pm 0.4$ & $8.6 \pm 0.8$ & $3 \pm 0.7$ & $3.33 \pm 0.4$ & $3 \pm 0.7$ \\
\hline & Class 3 & $1.33 \pm 0.4$ & $12 \pm 0.7$ & $3.6 \pm 0.4$ & $4.66 \pm 0.4$ & $1.33 \pm 0.8$ & $2.66 \pm 0.4$ \\
\hline & Class 4 & $2.33 \pm 0.4$ & $12.3 \pm 0.8$ & $5.3 \pm 0.8$ & $3.33 \pm 0.4$ & $0.33 \pm 0.4$ & $3 \pm 0.7$ \\
\hline & TCS & $23.29 \pm 4$ & $125.31 \pm 7.1$ & $71.2 \pm 6.7$ & $33.3 \pm 6.4$ & $33.3 \pm 6.4$ & $29.3 \pm 5.8$ \\
\hline
\end{tabular}

$\mathrm{A}=$ pure feed, $\mathrm{B}=$ OTA contaminated feed $(400 \mu \mathrm{g}$ of OTA $/ \mathrm{kg}$ of feed), $\mathrm{C}=$ OTA contaminated feed $(400 \mu \mathrm{g}$ of OTA $/ \mathrm{kg}$ of feed $)+0.3 \%$ Adsorbent, $\mathrm{D}=0 \mathrm{OTA}$ contaminated feed $(400 \mu \mathrm{g}$ of OTA $/ \mathrm{kg}$ of feed $)+0.6 \%$ Adsorbent, $E=$ OTA contaminated feed $(400 \mu \mathrm{g}$ of OTA $/ \mathrm{kg}$ of feed $)+0.9 \%$ Adsorbent, F $=$ OTA contaminated feed (400 $\mu \mathrm{g}$ of OTA $/ \mathrm{kg}$ of feed $)+1.2 \%$ Adsorbent.

\section{Histopathological observations of kidney and liver}

Histopathological observations of kidney and liver ( $6^{\text {th }}$ week) have been presented in (figure 3 and 4 : The images of the $1^{\text {st }}$ to the $5^{\text {th }}$ week has not been shown; only observations has been provided). Normal histological patterns with clear glomerular spaces and well conserved tubular structure were shown by the kidneys of chickens in group $A$ throughout the experimental period. Liver also showed normal histological structure in all the six weeks of the experiment. Hepatocytes had centrally placed nuclei with normal cytoplasm and the lobular structure of liver was retained.
The kidneys of the chicken in group B showed a typical histological pattern with slight grade of congestion at some places during the second week of the experiment. The pathological effect of ochratoxin was prominent at the end of the $4^{\text {th }}$ week because some areas of the kidney showed large size nuclei with irreversible condensed DNA in epithelial cells of renal tubules. Urinary spaces were occupied by proliferation of the epithelial cells in glomeruli and marked congestion was noted. Similarly, hepatocytes also showed moderate type of fatty changes. In some areas of the liver section mild type of cellular infiltration was observed around the blood vessels. Moderate degree of swellings and necrosis of hepatocytes was present at most of the 


\section{In Vivo Detoxification of Ochratoxin A by Highly Porous Magnetic Nanocomposites Prepared from Coconut Shell}

Table 3s $-3^{\text {rd }}$ week

\begin{tabular}{|c|c|c|c|c|c|c|c|}
\hline & & & & Group & & & \\
\hline & & & Ochra & A $400 \mu \mathrm{g} / \mathrm{kg} \mathrm{f}$ & & & \\
\hline & Comet class and TCS & $A$ & $\mathrm{~B}$ & $C$ & $\mathrm{D}$ & $E$ & $\mathrm{~F}$ \\
\hline & Class 0 & $91 \pm 1.2$ & $35.66 \pm 1.4$ & $63 \pm 0.7$ & $64.6 \pm 0.4$ & $72.3 \pm 4.2$ & $89.66 \pm 0.4$ \\
\hline & Class 1 & $2.66 \pm 0.4$ & $14.6 \pm 1.0$ & $22.66 \pm 0.8$ & $24.33 \pm 0.4$ & $25.3 \pm 4.6$ & $2.66 \pm 0.4$ \\
\hline Blond & Class 2 & $2.33 \pm 0.4$ & $13.66 \pm 0.8$ & $5 \pm 0.7$ & $3 \pm 0.7$ & $1.3 \pm 0.4$ & $2.33 \pm 0.4$ \\
\hline Blood & Class 3 & $2.66 \pm 0.4$ & $12.66 \pm 0.4$ & $4 \pm 0.7$ & $4.33 \pm 0.4$ & $0.6 \pm 0.4$ & $1.6 \pm 0.4$ \\
\hline & Class 4 & $1.33 \pm 0.4$ & $23.33 \pm 0.8$ & $5.33 \pm 0.4$ & $3.66 \pm 0.4$ & $0.3 \pm 0.4$ & $2.3 \pm 0.4$ \\
\hline & TCS & $20.62 \pm 4$ & $173.28 \pm 7$ & $65.98 \pm 5.9$ & $57.96 \pm 4.6$ & $31.29 \pm 8.2$ & $21.6 \pm 4$ \\
\hline & Class 0 & $90.66 \pm 2.2$ & $34.33 \pm 0.4$ & $57.66 \pm 2.4$ & $64 \pm 1.8$ & $70.3 \pm 0.4$ & $90.3 \pm 1.0$ \\
\hline & Class 1 & $2.33 \pm 0.8$ & $14.66 \pm 0.4$ & $24 \pm 1.4$ & $25.66 \pm 1.0$ & $22.66 \pm 0.8$ & $3.3 \pm 0.4$ \\
\hline liver & Class 2 & $2.33 \pm 0.4$ & $14.66 \pm 0.8$ & $5 \pm 0.7$ & $3.3 \pm 0.8$ & $2.3 \pm 0.4$ & $2.3 \pm 0.4$ \\
\hline Liver & Class 3 & $3 \pm 1.2$ & $13 \pm 0.7$ & $4.66 \pm 0.4$ & $4.6 \pm 0.4$ & $4 \pm 0.7$ & $2.3 \pm 0.4$ \\
\hline & Class 4 & $1.33 \pm 1.0$ & $23.3 \pm 0.4$ & $5.33 \pm 0.4$ & $4.3 \pm 0.4$ & $0.66 \pm 0.4$ & $1.6 \pm 0.4$ \\
\hline & TCS & $21.31 \pm 9.2$ & $176.3 \pm 5.7$ & $69.3 \pm 5.6$ & $63.62 \pm 5.4$ & $41.9 \pm 5.3$ & $21.6 \pm 4$ \\
\hline & Class 0 & $90.66 \pm 3.8$ & $32 \pm 0.7$ & $61.3 \pm 0.4$ & $60.3 \pm 0.4$ & $68 \pm 0.70$ & $89.6 \pm 0.8$ \\
\hline & Class 1 & $2 \pm 0.7$ & $15.66 \pm 0.4$ & $23.33 \pm 0.81$ & $26.6 \pm 0.8$ & $23.6 \pm 0.4$ & $3.3 \pm 0.8$ \\
\hline Kidnv & Class 2 & $2.66 \pm 0.4$ & $15 \pm 0.7$ & $5.3 \pm 0.4$ & $4 \pm 0.7$ & $3.6 \pm 0.4$ & $2.66 \pm 0.4$ \\
\hline Kidny & Class 3 & $2.33 \pm 1.7$ & $13.3 \pm 0.4$ & $4.3 \pm 0.4$ & $4.33 \pm 0.4$ & $4.3 \pm 0.4$ & $1.33 \pm 0.4$ \\
\hline & Class 4 & $2.33 \pm 0.4$ & $24 \pm 0.7$ & $5.6 \pm 0.4$ & $4.66 \pm 0.4$ & $0.3 \pm 0.4$ & $3 \pm 0$ \\
\hline & TCS & $24.62 \pm 8.2$ & $181.6 \pm 5.8$ & $69.6 \pm 4.4$ & $66.29 \pm 5$ & $45.29 \pm 4$ & $24.64 \pm 2.8$ \\
\hline
\end{tabular}

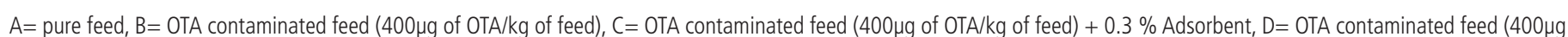

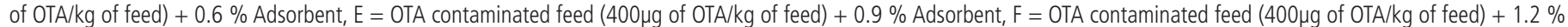
Adsorbent.

Table 4s $-4^{\text {th }}$ week

Group

\begin{tabular}{|c|c|c|c|c|c|c|c|}
\hline & \multicolumn{7}{|c|}{ Ochratoxin A $400 \mu \mathrm{g} / \mathrm{kg}$ feed } \\
\hline & Comet class and TCS & $A$ & $\mathrm{~B}$ & $\mathrm{C}$ & $\mathrm{D}$ & $\mathrm{E}$ & $\mathrm{F}$ \\
\hline \multirow{6}{*}{ Blood } & Class 0 & $90 \pm 4.3$ & $28.6 \pm 0.8$ & $64.33 \pm 0.4$ & $64.33 \pm 1.0$ & $68 \pm 1.2$ & $91 \pm 0.7$ \\
\hline & Class 1 & $2 \pm 0.7$ & $16.3 \pm 0.4$ & $18 \pm 0.7$ & $24.33 \pm 0.4$ & $26 \pm 1.2$ & $2.3 \pm 0.4$ \\
\hline & Class 2 & $1.66 \pm 0.8$ & $15.6 \pm 0.8$ & $6.33 \pm 0.4$ & $4.33 \pm 1.7$ & $5 \pm 0.7$ & $1.3 \pm 0.8$ \\
\hline & Class 3 & $2 \pm 0.7$ & $13 \pm 0.7$ & $5.66 \pm 0.4$ & $3.33 \pm 0.4$ & $0.66 \pm 0.4$ & $2.6 \pm 0.8$ \\
\hline & Class 4 & $2.66 \pm 0.4$ & $26.3 \pm 0.4$ & $5.66 \pm 0.4$ & $3.66 \pm 0.4$ & $0.33 \pm 0.4$ & $2.6 \pm 0.4$ \\
\hline & TCS & $21.96 \pm 6$ & $191.9 \pm 5.7$ & $70.28 \pm 4.3$ & $57.62 \pm 6.6$ & $39.3 \pm 5.4$ & $23.3 \pm 6$ \\
\hline \multirow{6}{*}{ Liver } & Class 0 & $90 \pm 0.7$ & $28.6 \pm 0.4$ & $63.33 \pm 1.4$ & $63.33 \pm 1.4$ & $68.3 \pm 0.8$ & $93.3 \pm 1.0$ \\
\hline & Class 1 & $4 \pm 0.7$ & $15.3 \pm 0.4$ & $18 \pm 0.7$ & $25.33 \pm 0.8$ & $25 \pm 0.7$ & $1.6 \pm 0.8$ \\
\hline & Class 2 & $1.33 \pm 0.4$ & $14 \pm 0.7$ & $6.66 \pm 1.0$ & $4 \pm 0.7$ & $5.33 \pm 0.4$ & $2.6 \pm 0.4$ \\
\hline & Class 3 & $2.33 \pm 0.4$ & $13.3 \pm 0.4$ & $5 \pm 0.7$ & $3.33 \pm 0.4$ & $0.66 \pm 0.4$ & $3 \pm 0.7$ \\
\hline & Class 4 & $2.33 \pm 0.4$ & $28.6 \pm 0.4$ & $6.33 \pm 0.4$ & $4 \pm 0.7$ & $0.66 \pm 0.4$ & $2 \pm 0.7$ \\
\hline & TCS & $22.97 \pm 4.3$ & $197.9 \pm 4.6$ & $71.64 \pm 6.4$ & $59.32 \pm 6.2$ & $40.2 \pm 4.3$ & $23.9 \pm 6.5$ \\
\hline \multirow{6}{*}{ Kidny } & Class 0 & $89.33 \pm 0.4$ & $23.6 \pm 2.6$ & $61.3 \pm 8.3$ & $62.33 \pm 1.0$ & $67.33 \pm 1.0$ & $89 \pm 0.7$ \\
\hline & Class 1 & $3 \pm 0.7$ & $18.6 \pm 0.81$ & $16.6 \pm 1.0$ & $26 \pm 1.2$ & $25 \pm 0.7$ & $3 \pm 0.70$ \\
\hline & Class 2 & $2.33 \pm 0.4$ & $16 \pm 0.7$ & $7.66 \pm 1.0$ & $4 \pm 0.7$ & $5.66 \pm 0.8$ & $2.3 \pm 0.4$ \\
\hline & Class 3 & $2.66 \pm 0.4$ & $13 \pm 0.7$ & $5.33 \pm 0.4$ & $3.33 \pm 0.4$ & $1.33 \pm 0.8$ & $3.3 \pm 0.4$ \\
\hline & Class 4 & $2.66 \pm 0.4$ & $30 \pm 0.7$ & $6.33 \pm 0.4$ & $4.33 \pm 0.4$ & $0.66 \pm 0.4$ & $2.3 \pm 0.4$ \\
\hline & TCS & $26.28 \pm 4.3$ & $209.6 \pm 7.1$ & $73.29 \pm 5.8$ & $61.31 \pm 5.4$ & $42.9 \pm 6.3$ & 26.94 .3 \\
\hline
\end{tabular}

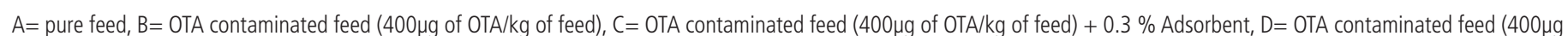

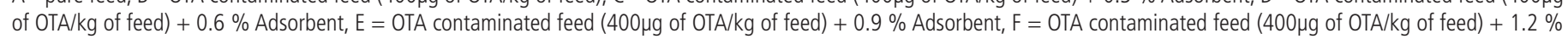
Adsorbent.

places. During the $6^{\text {th }}$ week of the experimental period, the kidneys as well as the livers showed severe types of pathological effects. Proliferation of the epithelial cells in glomeruli with hyalinization had reached its maximum degree. Extreme congestion was shown by the cells of renal tubules that has narrowed or blocked the lumen entirely. Similarly, liver also showed severe pathological effects, during the last week of the_experimental period that included acute cellular swelling of hepatocytes, congestion, unclear sinusoidal spaces and cell swelling. Individual cell necrosis was present, indicated by pyknosis in the hepatocytes. 


\section{In Vivo Detoxification of Ochratoxin A by Highly Porous Magnetic Nanocomposites Prepared from Coconut Shell}

Table 5s $-5^{\text {th }}$ week

\begin{tabular}{|c|c|c|c|c|c|c|c|}
\hline & \multicolumn{7}{|c|}{ Group } \\
\hline & \multicolumn{7}{|c|}{ Ochratoxin A $400 \mu \mathrm{g} / \mathrm{kg}$ feed } \\
\hline & Comet class and TCS & A & $\mathrm{B}$ & $C$ & $\mathrm{D}$ & $E$ & $\mathrm{~F}$ \\
\hline \multirow{6}{*}{ Blood } & Class 0 & $88 \pm 0.7$ & $23.66 \pm 2.1$ & $60 \pm 3.24$ & $64 \pm 3.53$ & $69 \pm 3.6$ & $87.33 \pm 1.4$ \\
\hline & Class 1 & $7.66 \pm 0.4$ & $11 \pm 0.7$ & $22.33 \pm 0.4$ & $27.66 \pm 1.4$ & $28.33 \pm 2.0$ & $6.33 \pm 0.4$ \\
\hline & Class 2 & $2.66 \pm 0.4$ & $16.33 \pm 1.0$ & $7.33 \pm 0.8$ & $4.33 \pm 1.0$ & $0.66 \pm 0.4$ & $2.33 \pm 0.4$ \\
\hline & Class 3 & $1.33 \pm 0.4$ & $18.33 \pm 1.0$ & $3.33 \pm 0.4$ & $3.33 \pm 0.4$ & $1.33 \pm 0.8$ & $2.66 \pm 0.4$ \\
\hline & Class 4 & $0.33 \pm 0.4$ & $30.66 \pm 1.0$ & $6.66 \pm 0.4$ & $3.6 \pm 0.4$ & $0.66 \pm 0.4$ & $0.66 \pm 0.4$ \\
\hline & TCS & $18.29 \pm 4$ & $221.29 \pm 9.7$ & $73.62 \pm 4.8$ & $60.95 \pm 6.2$ & $36.28 \pm 6.8$ & $21.62 \pm 4$ \\
\hline \multirow{6}{*}{ Liver } & Class 0 & $87 \pm 0.7$ & $18 \pm 3.9$ & $57.66 \pm 0.4$ & $60 \pm 3.6$ & $69.33 \pm 2.9$ & $87 \pm 0.7$ \\
\hline & Class 1 & $7.66 \pm 0.4$ & $12.33 \pm 1.0$ & $25 \pm 1.2$ & $28.33 \pm 2.9$ & $26.66 \pm 1.6$ & $7.66 \pm 0.4$ \\
\hline & Class 2 & $2.33 \pm 0.4$ & $18 \pm 0.7$ & $7 \pm 0.7$ & $4 \pm 0.7$ & $2.33 \pm 0.8$ & $2.33 \pm 0.8$ \\
\hline & Class 3 & $2.66 \pm 0.4$ & $19.33 \pm 0.8$ & $3 \pm 0.7$ & $3.33 \pm 0.8$ & $0.66 \pm 0.4$ & $2.33 \pm 0.4$ \\
\hline & Class 4 & $0.33 \pm 0.4$ & $30.66 \pm 1.0$ & $7.33 \pm 0.8$ & $4 \pm 0.7$ & $1 \pm 0.7$ & $1.66 \pm 0.4$ \\
\hline & TCS & $21.62 \pm 4$ & $228.96 \pm 8.8$ & $77.32 \pm 7.9$ & $62.32 \pm 9.5$ & $37.3 \pm 7.2$ & $25.95 \pm 4.8$ \\
\hline \multirow{6}{*}{ Kidny } & Class 0 & $86 \pm 1.8$ & $19.66 \pm 1.4$ & $55.33 \pm 3.8$ & $59.66 \pm 1.0$ & $66.33 \pm 1.4$ & $86.33 \pm 1.0$ \\
\hline & Class 1 & $7.66 \pm 0.4$ & $11 \pm 0.7$ & $27 \pm 1.2$ & $28.33 \pm 1.0$ & $31 \pm 1.8$ & $6.33 \pm 0.4$ \\
\hline & Class 2 & $2.33 \pm 0.8$ & $18 \pm 0.7$ & $7 \pm 0.7$ & $4.33 \pm 0.4$ & $0.66 \pm 0.8$ & $3 \pm 0.7$ \\
\hline & Class 3 & $2.66 \pm 0.4$ & $19.66 \pm 0.8$ & $4.33 \pm 0.4$ & $3.66 \pm 0.4$ & $1.66 \pm 0.4$ & $2.66 \pm 0.4$ \\
\hline & Class 4 & $1.33 \pm 0.4$ & $31.66 \pm 1.0$ & $7 \pm 0.7$ & $4 \pm 0$ & $0.33 \pm 0.4$ & $1.66 \pm 0.4$ \\
\hline & TCS & $25.62 \pm 4.8$ & $232.62 \pm 8.5$ & $81.99 \pm 6.6$ & $63.97 \pm 3$ & $38.6 \pm 6.2$ & $26.95 \pm 4.6$ \\
\hline
\end{tabular}

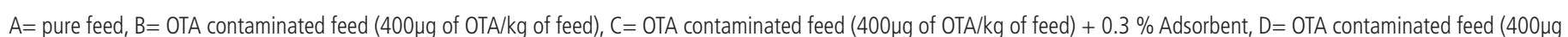

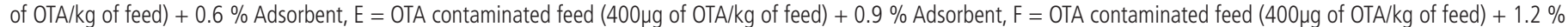
Adsorbent.

Table $6 s-6^{\text {th }}$ week

Group

\begin{tabular}{|c|c|c|c|c|c|c|c|}
\hline & \multicolumn{7}{|c|}{ Ochratoxin A 400 $\mu \mathrm{g} / \mathrm{kg}$ feed } \\
\hline & Comet class and TCS & A & B & $\mathrm{C}$ & D & $\mathrm{E}$ & $\mathrm{F}$ \\
\hline \multirow{6}{*}{ Blood } & Class 0 & $90 \pm 0.7$ & $23 \pm 2.1$ & $56.3 \pm 2.1$ & $62 \pm 2.4$ & $64.3 \pm 3.6$ & $90 \pm 2.4$ \\
\hline & Class 1 & $3 \pm 1.2$ & $11.3 \pm 0.8$ & $27 \pm 1.8$ & $25 \pm 3.0$ & $30.66 \pm 2.8$ & $1.66 \pm 0.8$ \\
\hline & Class 2 & $1.66 \pm 0.4$ & $17 \pm 0.7$ & $6 \pm 0.7$ & $4.33 \pm 0.8$ & $4.33 \pm 1.0$ & $2.66 \pm 0.4$ \\
\hline & Class 3 & $2.66 \pm 0.4$ & $18 \pm 0.7$ & $5 \pm 0.7$ & $4.66 \pm 0.4$ & $0.66 \pm 0.4$ & $2.66 \pm 0.8$ \\
\hline & Class 4 & $2.66 \pm 0.4$ & $30.66 \pm 1.4$ & $5.66 \pm 0.8$ & $4 \pm 0.7$ & $0.33 \pm 0.4$ & $3 \pm 1.8$ \\
\hline & TCS & $24.94 \pm 4$ & $221.97 \pm 9.9$ & $76.64 \pm 8.5$ & $63.64 \pm 8.6$ & $43.06 \pm 7.6$ & $26.96 \pm 11.2$ \\
\hline \multirow{6}{*}{ Liver } & Class 0 & $89.33 \pm 0.4$ & $22.33 \pm 0.8$ & $56.33 \pm 1.08$ & $61.66 \pm 4.9$ & $64.66 \pm 1.0$ & $89.33 \pm 1.0$ \\
\hline & Class 1 & $2.66 \pm 0.4$ & $11.33 \pm 0.4$ & $25.66 \pm 0.8$ & $26 \pm 3.7$ & $29.33 \pm 0.4$ & $2.66 \pm 0.8$ \\
\hline & Class 2 & $2.33 \pm 0.4$ & $16.33 \pm 0.4$ & $5.66 \pm 2.8$ & $4 \pm 1.4$ & $4.33 \pm 1.0$ & $1.66 \pm 0.8$ \\
\hline & Class 3 & $3 \pm 0.7$ & $18 \pm 0.7$ & $5 \pm 1.2$ & $4.66 \pm 0.8$ & $0.66 \pm 0.8$ & $2.66 \pm 0.4$ \\
\hline & Class 4 & $2.66 \pm 0.4$ & $32 \pm 0.7$ & $7.33 \pm 0.4$ & $4.33 \pm 2.1$ & $1 \pm 4.8$ & $3.66 \pm 0.4$ \\
\hline & TCS & $26.96 \pm 4.9$ & $225.99 \pm 6.1$ & $81.3 \pm 11.6$ & $65.3 \pm 17.3$ & $43.97 \pm 6.4$ & $27.61 \pm 5.2$ \\
\hline \multirow{6}{*}{ Kidny } & Class 0 & $89 \pm 1.8$ & $19 \pm 1.2$ & $53.33 \pm 2.1$ & $60 \pm 3.7$ & $66.33 \pm 1.4$ & $88.66 \pm 0.8$ \\
\hline & Class 1 & $2.66 \pm 0.4$ & $11 \pm 0.7$ & $25.66 \pm 0.8$ & $27 \pm 1.8$ & $25.33 \pm 1.4$ & $2 \pm 0.7$ \\
\hline & Class 2 & $2.33 \pm 0.4$ & $18.33 \pm 1.0$ & $5.66 \pm 0.4$ & $4 \pm 1.2$ & $5.33 \pm 0.4$ & $2 \pm 0$ \\
\hline & Class 3 & $3 \pm 0.7$ & $18.66 \pm 0.8$ & $5.33 \pm 0.8$ & $4.33 \pm 0.4$ & $1.33 \pm 0.4$ & $3.33 \pm 0.4$ \\
\hline & Class 4 & $3 \pm 0.7$ & $33 \pm 0.7$ & $7.33 \pm 0.4$ & $4.66 \pm 0.8$ & $1 \pm 0$ & $4 \pm 0.7$ \\
\hline & TCS & $28.32 \pm 6.1$ & $235.64 \pm 7.9$ & $82.29 \pm 5.6$ & $66.63 \pm 8.6$ & $43.98 \pm 3.4$ & $31.99 \pm 4.7$ \\
\hline
\end{tabular}

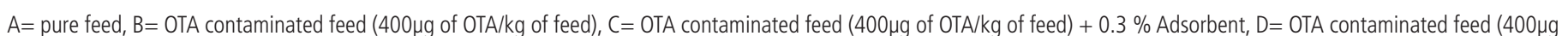

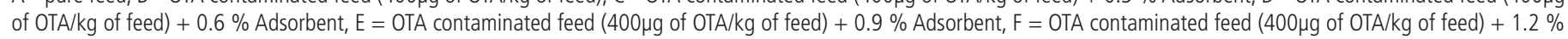
Adsorbent.

The kidney and liver in groups (C, D, E and F) (treated with different concentrations of adsorbent) did not show any violation from the normal histological pattern during the first week of the experimental period. While Mild to moderate degrees of pathological changes were observed during the third and fourth week of the experimental period in groups $C$ and D of birds that were provided with 0.3 and $0.6 \%(\mathrm{w} / \mathrm{w})$ of prepared adsorbent mixed with their feed. These pathological changes include slight congestion in the epithelial cells of renal tubules, thickening of the bowman's capsules and larger nuclei 
Gulfam N, Zahoor M,

Khisroom M, Khan FA
In Vivo Detoxification of Ochratoxin A by Highly Porous Magnetic Nanocomposites Prepared from Coconut Shell
A

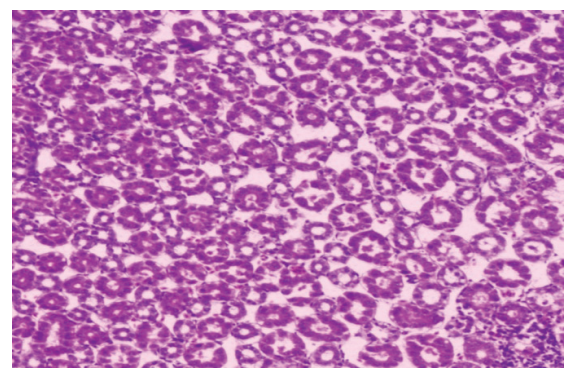

B

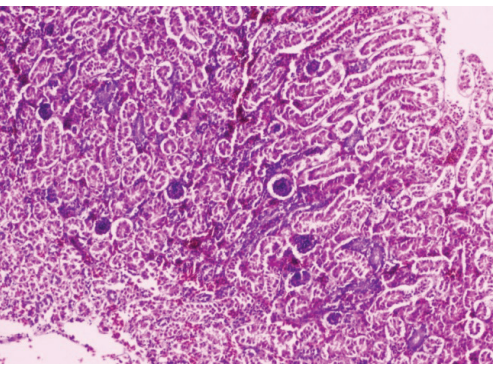

C

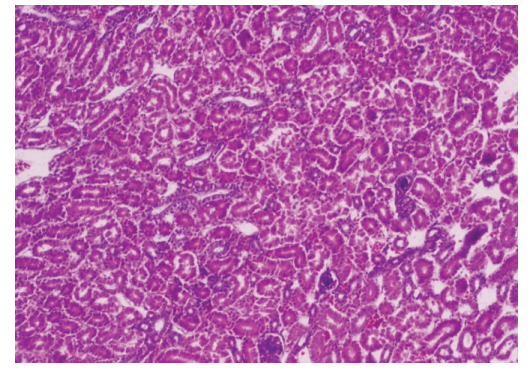

D

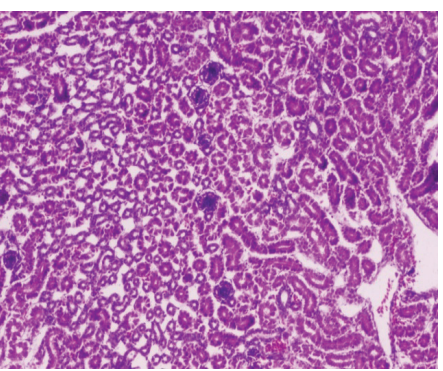

E
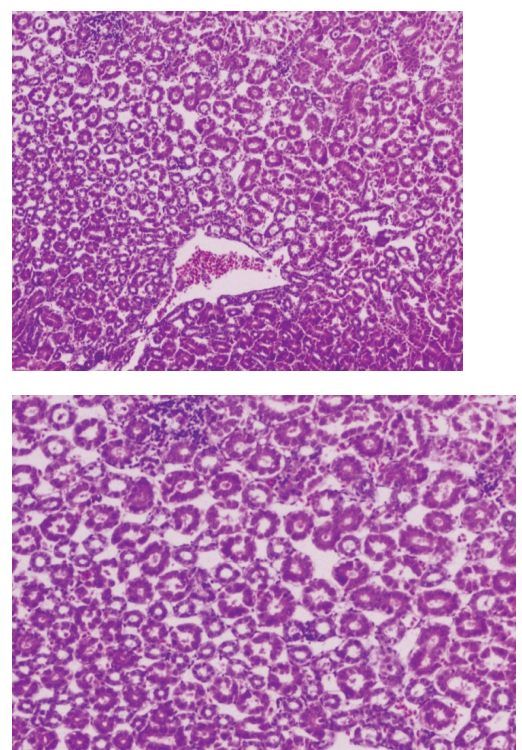

A

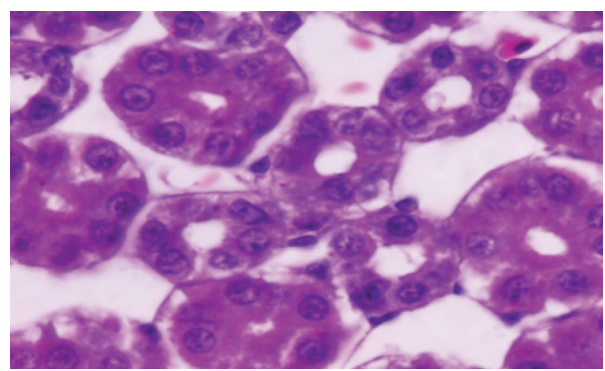

B

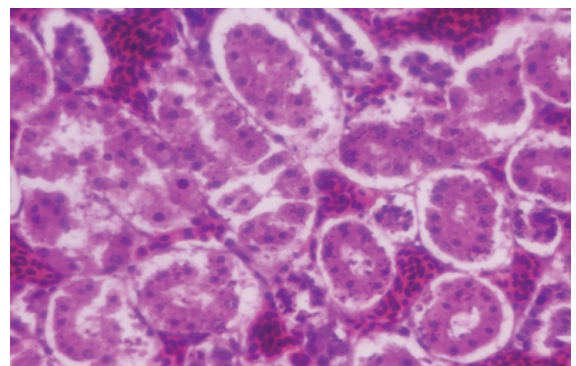

C

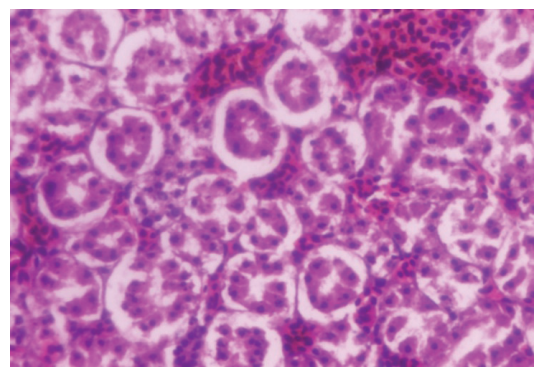

D

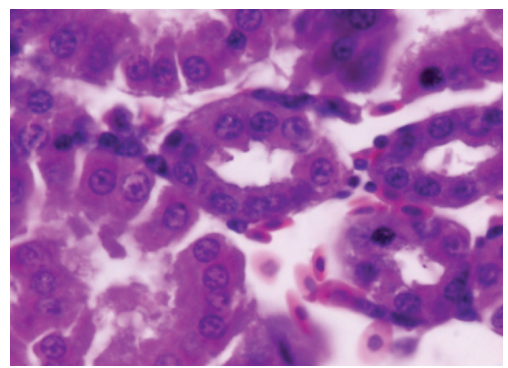

E

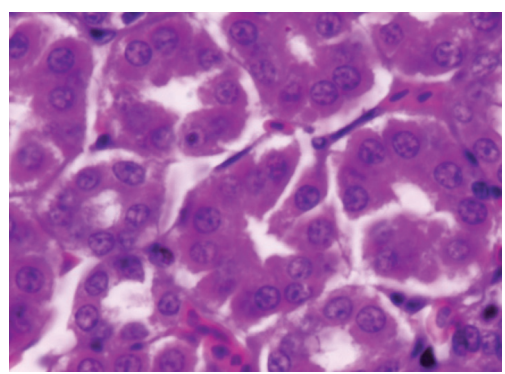

$\mathrm{F}$

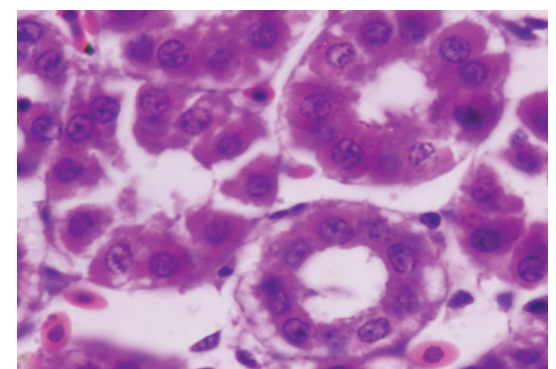

Figure 3 - Photomicrographs showing histopathology of kidney of chickens of 42 days of age in different groups at two different magnifications. 
Gulfam N, Zahoor M,

Khisroom M, Khan FA
In Vivo Detoxification of Ochratoxin A by Highly Porous Magnetic Nanocomposites Prepared from Coconut Shell
A

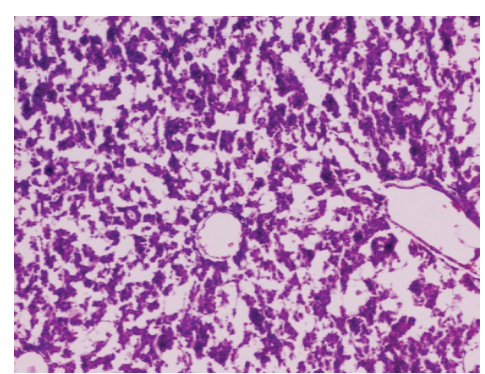

B

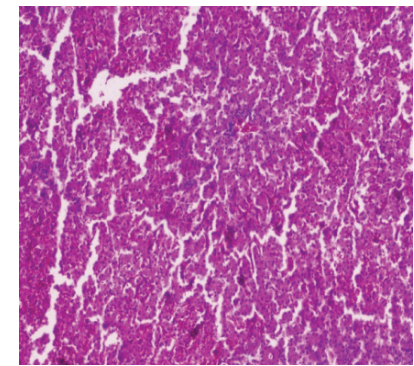

C

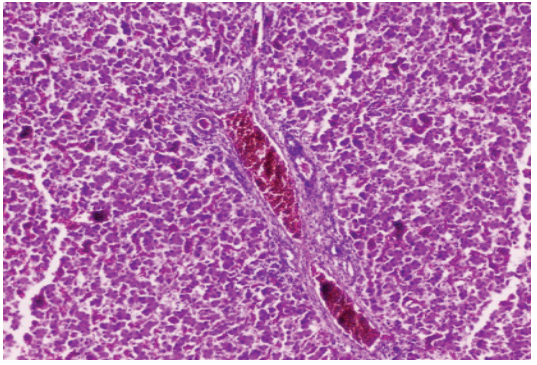

D

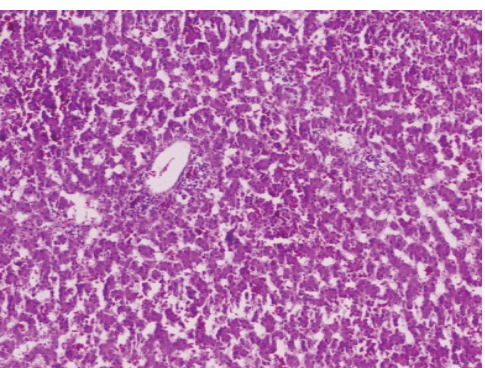

E

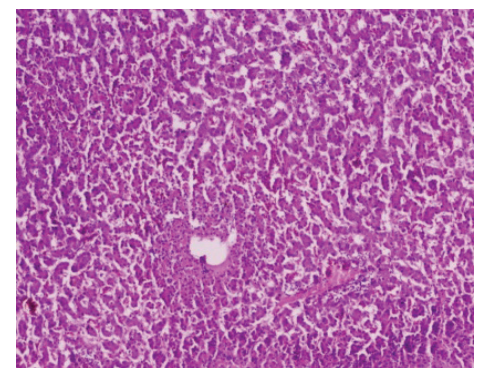

$\mathrm{F}$

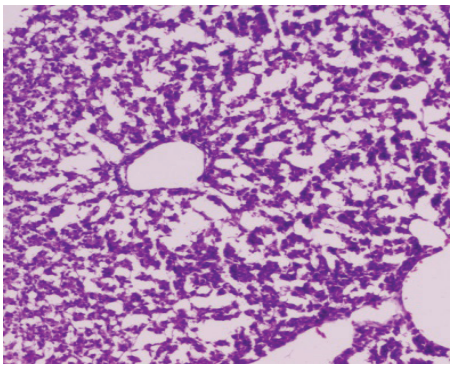

A

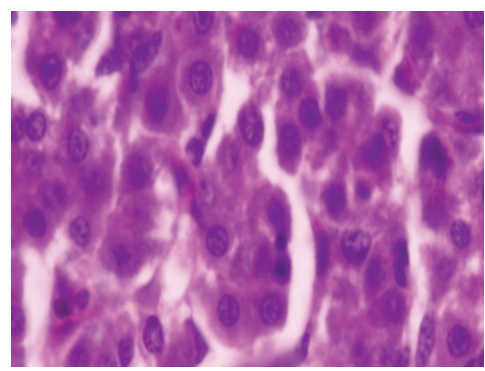

B

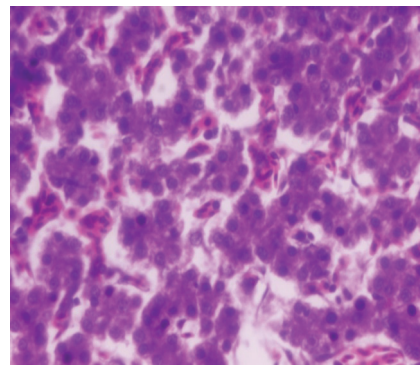

C

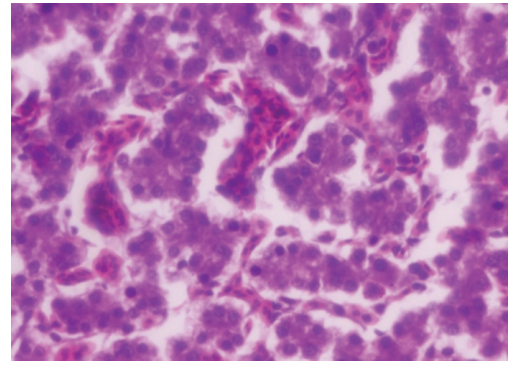

D

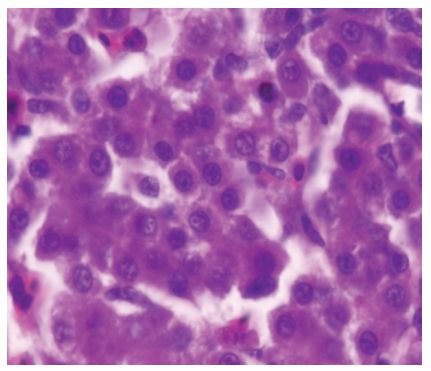

E

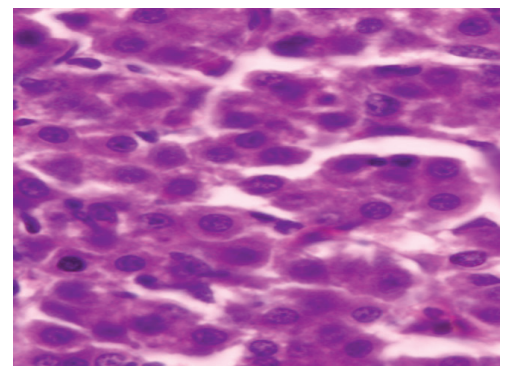

$\mathrm{F}$

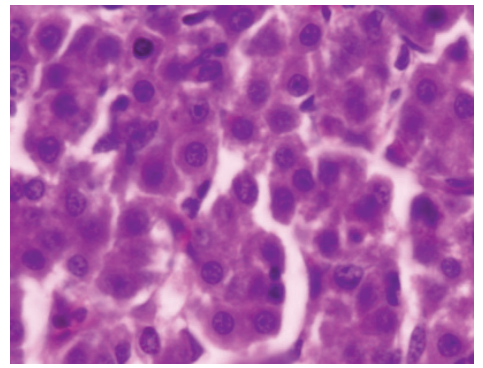

Figure 4 - Photomicrographs showing histopathology of the liver of different groups of chickens of 42 days of age, at two different magnifications. 
with irreversible condensed DNA in the epithelial cells of renal tubules. Individual hepatocytic necrosis and hepatocytes swelling of moderate degree was present at some places. Slight degree of cellular diffusion in the interstices around the blood vessels was observed in liver sections. No such pathological changes were observed in the kidney and liver samples in groups $E$ and $F$ of chicken that were kept on feed mixed with 0.9 and $1.2 \%(\mathrm{w} / \mathrm{w})$ of adsorbent derived from coconut shell. Microscopically, pathological changes observed in the kidneys and liver sections of broiler chicks, exposed to OTA contaminated feed resulted in congestion, necrotic and degenerative changes in the renal tubular epithelial cells. These complications were much prominent in positive controlled group (ochratoxin mixed feed without adsorbent) as compared to other groups. Similar observations regarding OTA induced nephropathy in avian have been reported by Kumar et al. (2004).

\section{Gross lesions and morphological alter- ations}

Table (7s) and figure 5 and 6 represent the score of gross lesions in various organs of poultry chicken (1st week to the $6^{\text {th }}$ week). Chickens in group $A$ and
F showed normal morphology of different organs throughout the whole length of the experiment therefore, no gross lesions were observed on kidney, liver and heart. Kidney and liver were normal in size, shape, texture and color. On the other hand, chicks in group B showed gross lesions on kidney and liver. These lesions appeared in the $2^{\text {nd }}$ week on the kidney and in the $3^{\text {rd }}$ week on the liver. The score and severity of gross lesions increased progressively and had reached its maximum during the sixth week of the experiment. Comparatively fewer lesions and morphological alterations were observed by chickens in group $C$ than that in group $B$. In case in group $D$ and $\mathrm{E}$, minor morphological alterations and very low score of lesions were recorded for kidney. Besides the results in group $D$ and $E$ were found very close to each other. Variation in color, size, texture and frequency of gross lesions in the liver and kidneys of chickens of different groups was subjectively scored by visual examination and has been presented in Table 7s.

Feeding of OTA to the birds resulted in edema and tubular necrosis particularly in proximal convoluted tubules (Hanif et al., 2006). Ortatatli et al. (2005) reported glomerulonephrosis, epithelial cells proliferation and multiple edemas like structures in the

Table 7s - Gross lesions and morphological alterations in different organs of broiler chickens kept on different concentrations of the prepared adsorbent derived from coconut shell.

\begin{tabular}{|c|c|c|c|c|c|c|c|c|}
\hline \multirow{3}{*}{$\begin{array}{l}\text { Time period } \\
\text { Week (days) }\end{array}$} & \multirow{3}{*}{ Organs } & \multirow{3}{*}{ Maximum possible score } & \multicolumn{6}{|c|}{ Groups } \\
\hline & & & \multicolumn{6}{|c|}{ Ochratoxin A 400 $\mu \mathrm{g} / \mathrm{kg}$ feed } \\
\hline & & & A & $B$ & C & $\mathrm{D}$ & $E$ & $\mathrm{~F}$ \\
\hline \multirow{3}{*}{ First week (7) days } & kidney & 9 & 0 & 0 & 0 & 0 & 0 & 0 \\
\hline & liver & 9 & 0 & 0 & 0 & 0 & 0 & 0 \\
\hline & heart & 9 & 0 & 0 & 0 & 0 & 0 & 0 \\
\hline \multirow[t]{2}{*}{ Cumulative score } & & 27 & 0 & 0 & 0 & 0 & 0 & 0 \\
\hline & kidney & 9 & 0 & 3 & 0 & 1 & 0 & 0 \\
\hline \multirow[t]{2}{*}{ second week (14) days } & liver & 9 & 0 & 0 & 0 & 0 & 0 & 0 \\
\hline & heart & 9 & 0 & 0 & 0 & 0 & 0 & 0 \\
\hline \multirow[t]{2}{*}{ Cumulative score } & & 27 & 0 & 3 & 0 & 1 & 0 & 0 \\
\hline & kidney & 9 & 0 & 5 & 1 & 0 & 0 & 0 \\
\hline \multirow[t]{2}{*}{ third week (21) days } & liver & 9 & 0 & 3 & 0 & 0 & 0 & 0 \\
\hline & heart & 9 & 0 & 0 & 0 & 0 & 0 & 0 \\
\hline \multirow[t]{2}{*}{ Cumulative score } & & 27 & 0 & 8 & 1 & 0 & 0 & 0 \\
\hline & kidney & 9 & 0 & 7 & 3 & 1 & 0 & 0 \\
\hline \multirow[t]{2}{*}{ Forth week (28) days } & liver & 9 & 0 & 3 & 1 & 0 & 0 & 0 \\
\hline & heart & 9 & 0 & 0 & 0 & 0 & 0 & 0 \\
\hline \multirow[t]{2}{*}{ Cumulative score } & & 27 & 0 & 10 & 4 & 1 & 0 & 0 \\
\hline & kidney & 9 & 0 & 8 & 3 & 1 & 0 & 0 \\
\hline \multirow[t]{2}{*}{ Fifth week (35) days } & liver & 9 & 0 & 4 & 1 & 0 & 0 & 0 \\
\hline & heart & 9 & 0 & 0 & 0 & 0 & 0 & 0 \\
\hline \multirow[t]{2}{*}{ Cumulative score } & & 27 & 0 & 12 & 4 & 1 & 0 & 0 \\
\hline & kidney & 9 & 0 & 8 & 3 & 1 & 1 & 0 \\
\hline \multirow[t]{2}{*}{ Sixth week (42) days } & liver & 9 & 0 & 5 & 2 & 0 & 0 & 0 \\
\hline & heart & 9 & 0 & 0 & 0 & 0 & 0 & 0 \\
\hline Cumulative score & & 27 & 0 & 13 & 5 & 1 & 1 & 0 \\
\hline
\end{tabular}


Gulfam N, Zahoor M,

Khisroom M, Khan FA

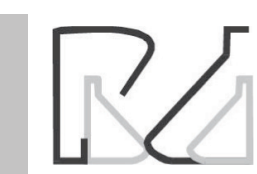

In Vivo Detoxification of Ochratoxin A by Highly Porous Magnetic Nanocomposites Prepared from Coconut Shell

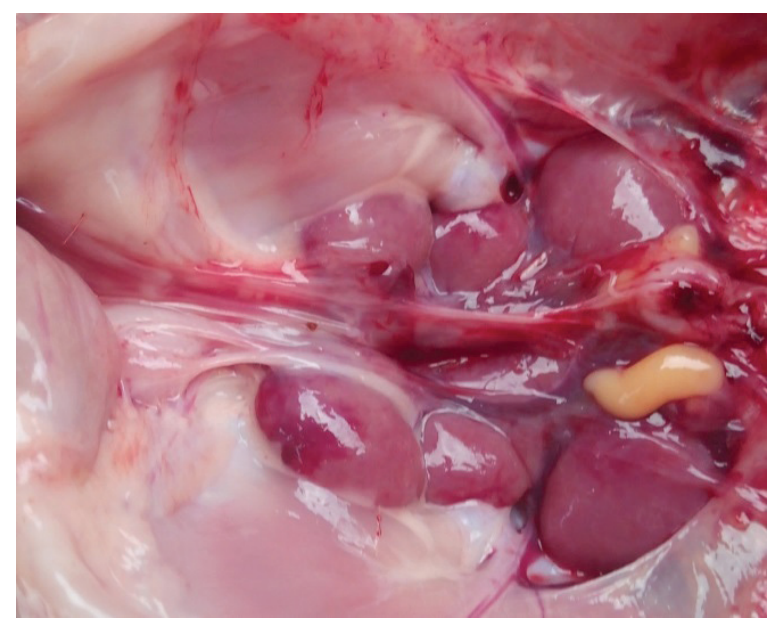

A

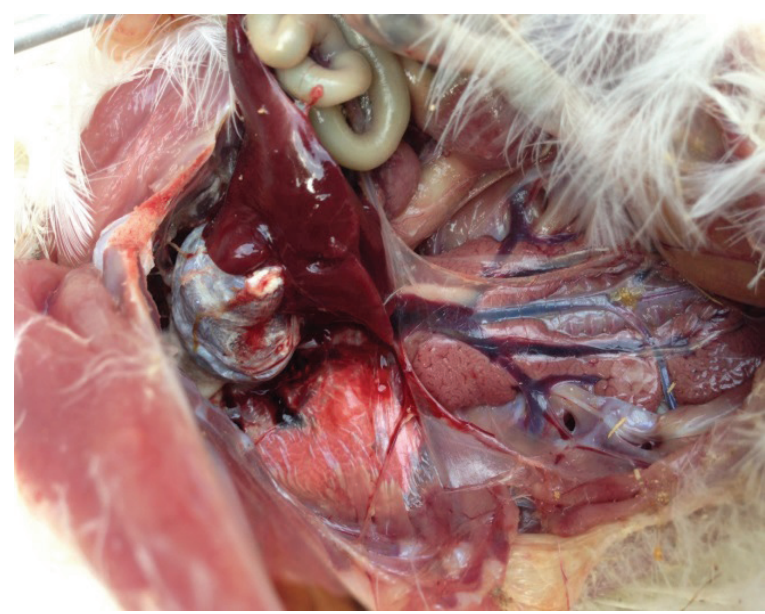

C

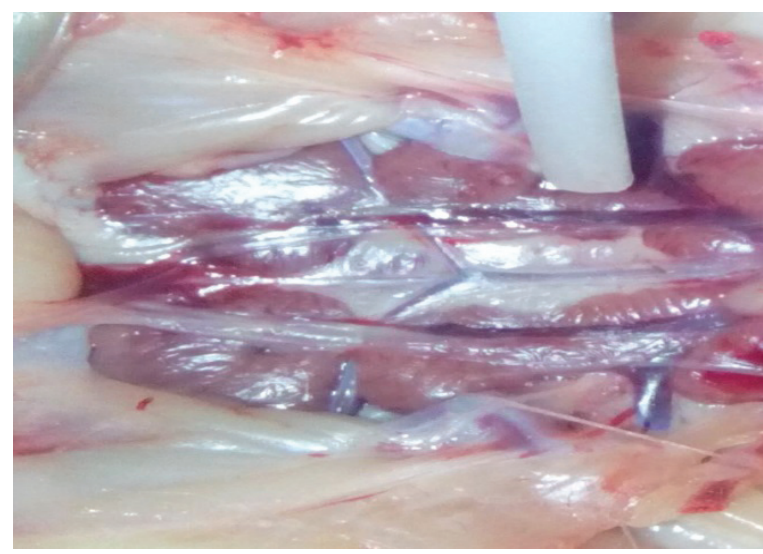

E

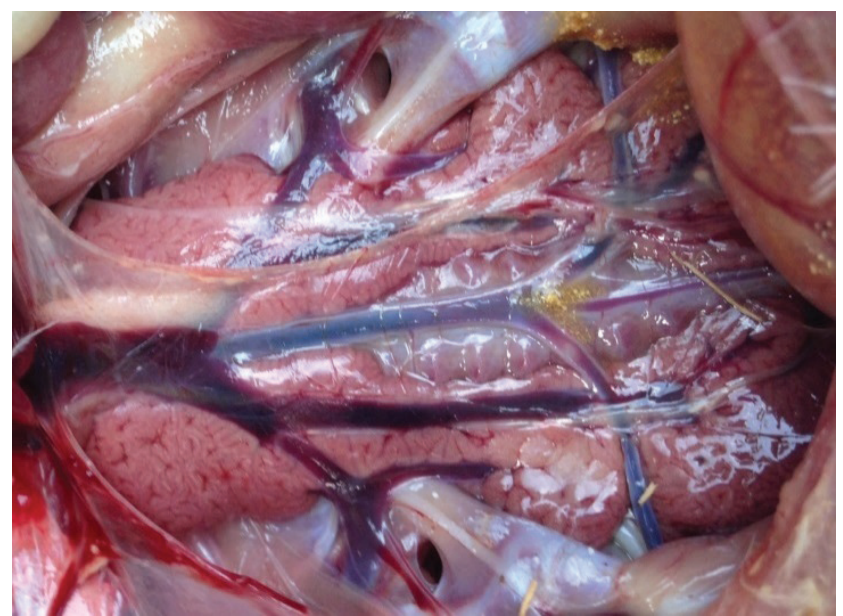

B

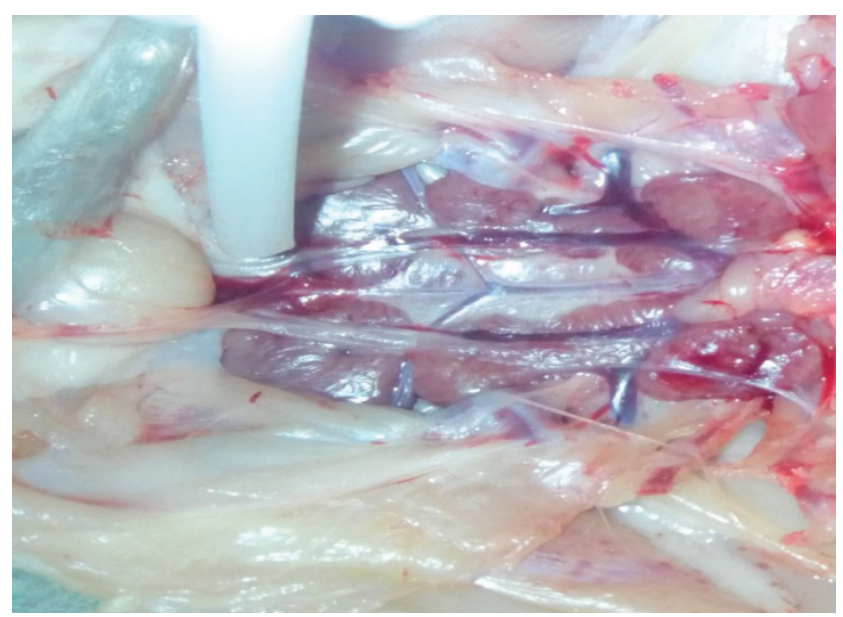

D

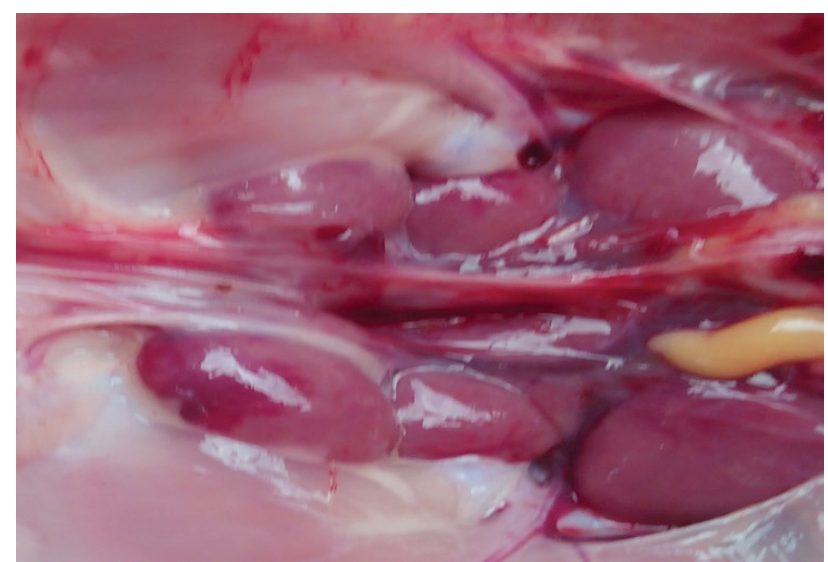

$\mathrm{F}$

Figure 5 - Photomicrographs showing kidney lesions in different groups.

kidneys parenchyma cells in chickens fed on OTA contaminated diet. The lesions observed in the liver and kidneys of chicks in group "B" sacrificed at the end of the experiment week, showed enlarged kidneys with prominent lesions. Also, pale friable liver with petechiae on the surface were also observed. Swollen kidneys and liver with pinpoint hemorrhages in laying hens and in broiler chicks have been described by several researchers (Kumar et al., 2004; Politis et al., 2005). Gross lesions on different organs in broiler chicks during ochratoxicosis have also been reported (Arbillaga et al., 2007). 


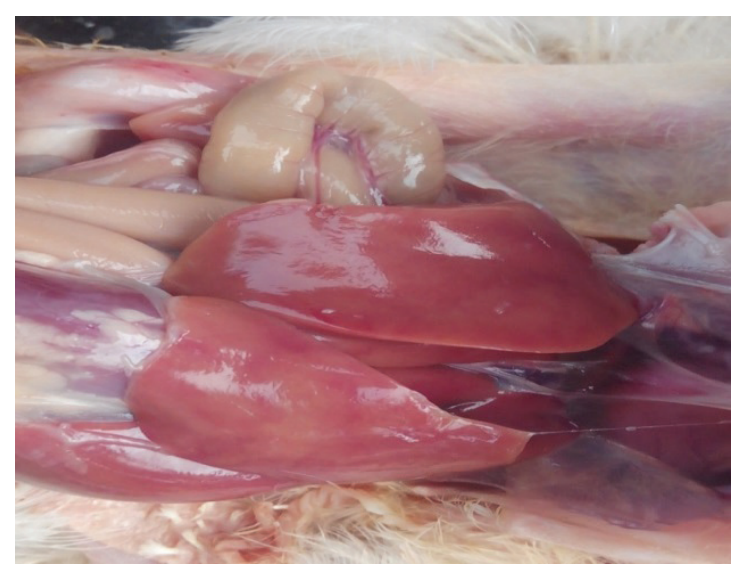

A

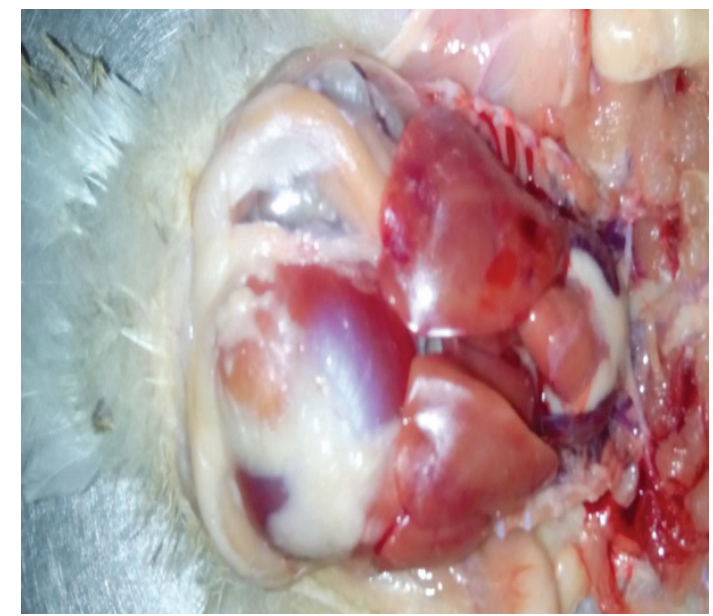

C

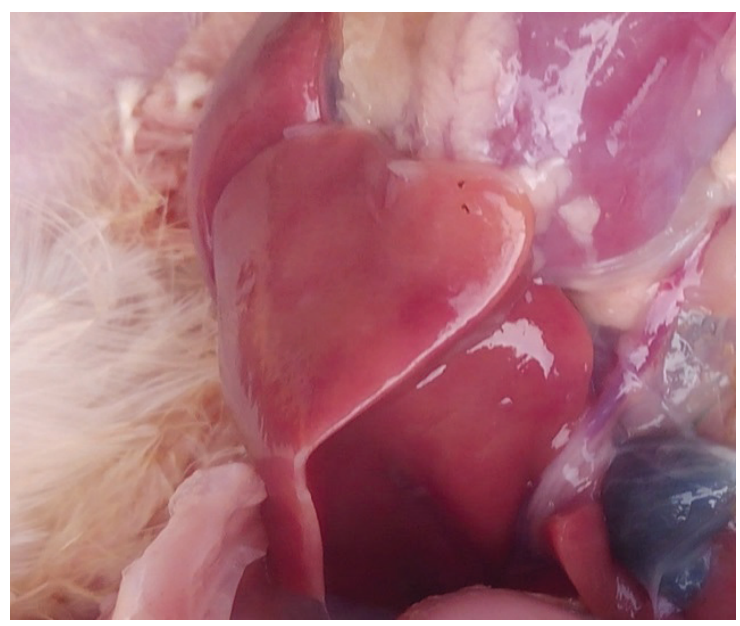

E

Figure 6 - Photomicrographs showing liver lesions in different groups.

\section{Enlargement of different organs}

Table 8s indicated relative enlargement of different organs of chicks during six weeks of the_experiment ( $1^{\text {st }}$ week to the $6^{\text {th }}$ week). Normal size and appearance of visceral organs were shown by chickens in group $A$,

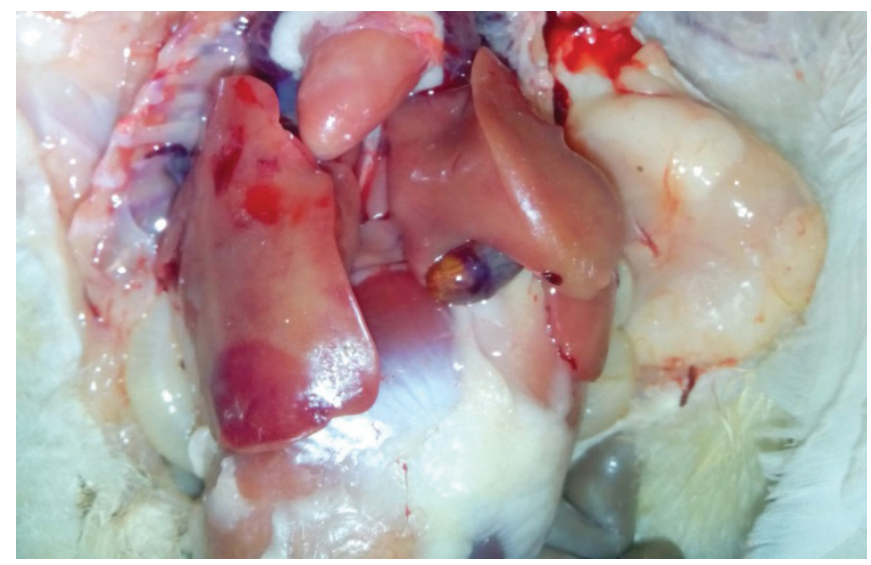

B

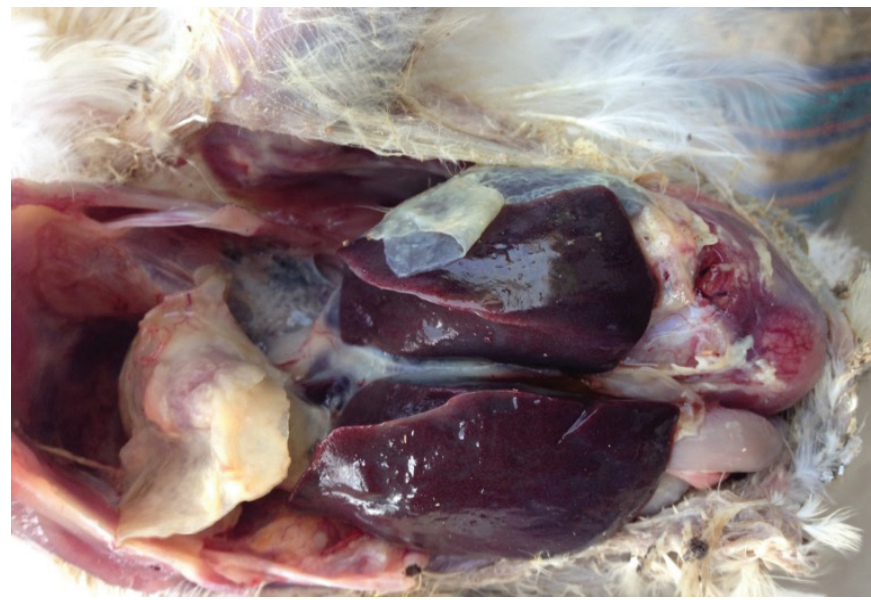

D

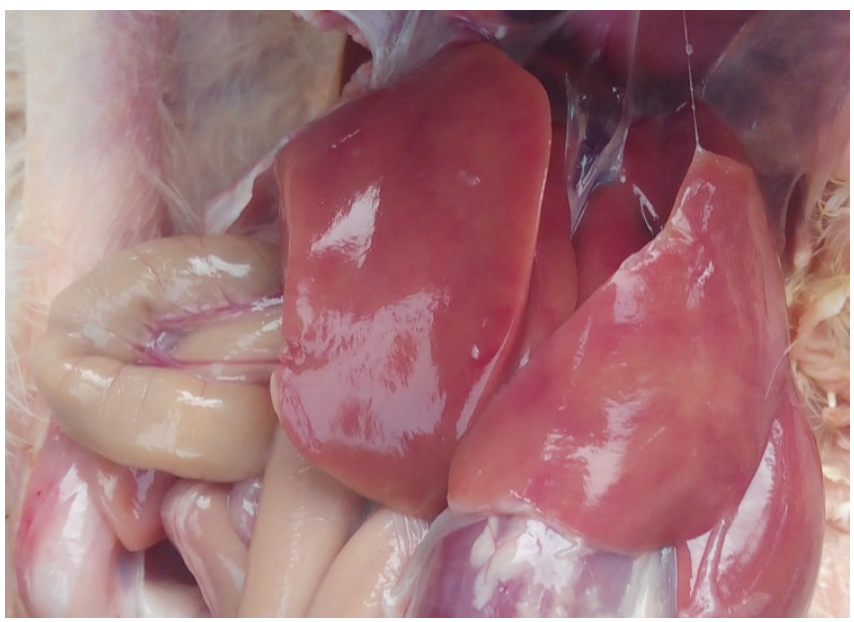

$\mathrm{F}$

$\mathrm{E}$ and $\mathrm{F}$ during the entire experimental duration. Under keen observation, the kidneys in group B showed slight enlargement during the $2^{\text {nd }}$ week, whereas the liver remained normal. Minor enlargement of the liver was shown by birds in group B during the third week of the experiment. The degree of enlargement of visceral 


\section{In Vivo Detoxification of Ochratoxin A by Highly Porous Magnetic Nanocomposites Prepared from Coconut Shell}

Table 8s - Enlargement of different organs of broiler chickens kept on different concentrations of the prepared adsorbent derived from coconut shell.

\begin{tabular}{|c|c|c|c|c|c|c|c|c|}
\hline \multirow{3}{*}{$\begin{array}{l}\text { Time period } \\
\text { Week(days) }\end{array}$} & \multirow{3}{*}{ Organs } & \multirow{3}{*}{ Maximum possible score } & \multicolumn{6}{|c|}{ Groups } \\
\hline & & & \multicolumn{6}{|c|}{ Ochratoxin A $400 \mu \mathrm{g} / \mathrm{kg}$ feed } \\
\hline & & & A & $B$ & C & $\mathrm{D}$ & $\mathrm{E}$ & $\mathrm{F}$ \\
\hline \multirow{3}{*}{ First week (7) days } & kidney & 9 & 0 & 0 & 0 & 0 & 0 & 0 \\
\hline & liver & 9 & 0 & 0 & 0 & 0 & 0 & 0 \\
\hline & heart & 9 & 0 & 0 & 0 & 0 & 0 & 0 \\
\hline \multirow[t]{2}{*}{ Cumulative score } & & 27 & 0 & 0 & 0 & 0 & 0 & 0 \\
\hline & kidney & 9 & 0 & 2 & 0 & 0 & 0 & 0 \\
\hline \multirow[t]{2}{*}{ second week (14) days } & liver & 9 & 0 & 0 & 0 & 0 & 0 & 0 \\
\hline & heart & 9 & 0 & 0 & 0 & 0 & 0 & 0 \\
\hline \multicolumn{2}{|l|}{ Cumulative score } & 27 & 0 & 2 & 0 & 0 & 0 & 0 \\
\hline \multirow{3}{*}{$\begin{array}{l}\text { third week } \\
(21) \text { days }\end{array}$} & kidney & 9 & 0 & 3 & 2 & 1 & 0 & 0 \\
\hline & liver & 9 & 0 & 1 & 0 & 0 & 0 & 0 \\
\hline & heart & 9 & 0 & 0 & 0 & 0 & 0 & 0 \\
\hline \multicolumn{2}{|l|}{ Cumulative score } & 27 & 0 & 4 & 2 & 1 & 0 & 0 \\
\hline \multirow{3}{*}{ Forth week (28) days } & kidney & 9 & 0 & 6 & 3 & 0 & 0 & 0 \\
\hline & liver & 9 & 0 & 2 & 1 & 0 & 0 & 0 \\
\hline & heart & 9 & 0 & 0 & 0 & 0 & 0 & 0 \\
\hline \multirow[t]{2}{*}{ Cumulative score } & & 27 & 0 & 8 & 4 & 0 & 0 & 0 \\
\hline & kidney & 9 & 0 & 6 & 3 & 1 & 0 & 0 \\
\hline \multirow[t]{2}{*}{ Fifth week (35) days } & liver & 9 & 0 & 3 & 1 & 0 & 0 & 0 \\
\hline & heart & 9 & 0 & 0 & 0 & 0 & 0 & 0 \\
\hline \multirow[t]{2}{*}{ Cumulative score } & & 27 & 0 & 9 & 4 & 1 & 0 & 0 \\
\hline & kidney & 9 & 0 & 7 & 3 & 1 & 0 & 0 \\
\hline \multirow[t]{2}{*}{ Sixth week (42) days } & liver & 9 & 0 & 2 & 1 & 0 & 0 & 0 \\
\hline & heart & 9 & 0 & 0 & 0 & 0 & 0 & 0 \\
\hline Cumulative score & & 27 & 0 & 9 & 4 & 1 & 0 & 0 \\
\hline
\end{tabular}

organs (kidney and liver) in group B increased with the increase in exposure duration of chicks to ochratoxin A contaminated feed. The kidneys in group C $(0.3 \%$ $\mathrm{w} / \mathrm{W})$ and $\mathrm{D}(0.6 \% \mathrm{w} / \mathrm{W})$ showed very low degree of enlargement when compared to group B. However, kidney, liver and heart in group $\mathrm{E}$ and $\mathrm{F}$ showed normal appearance and no enlargement were observed at all during the experiment. The sequence for overall enlargement of kidney and liver was in the following order: group $B>$ group $C>$ group $D>$ throughout the experimental weeks, while group $A, E$ and $F$ have shown normal size of these organs.

Kidney enlargements and moderate liver enlargement in intoxicated broiler chicks have also been documented by Verma et al. (2004) as well. It was concluded that the addition of different levels of adsorbent in ochratoxin A contaminated feed ameliorated the hazardous effects of ochratoxin A.

\section{Relative body weight}

Table 9s represented relative body weights of chicks kept on different concentrations of the prepared adsorbent derived from coconut shell ( $1^{\text {st }}$ week to the $6^{\text {th }}$ week). There was no significant difference in the body weight of chickens in group $A$ and $D$. Birds in group $A$ and $C$ presented a relative significant difference at the end of the $3^{\text {rd }}, 4^{\text {th }} 5^{\text {th }}$ and $6^{\text {th }}$ weeks. Similarly, group $C, D, E$ and $F$ also exhibited higher significant differences when compared to group $\mathrm{B}$. The maximum body weights were observed in group $F$ followed by group $E$ and $A$.

Early researchers have observed reduction in body weight gain of chicks when exposed to dietary ochratoxin A contaminated feed (Peckham et al., 1971; Kumar et al., 2004). Our study showed that body weight of chicks in group B decreased throughout the course of the study. The food conversion ratio (FCR) in group B was also impaired, whereas those groups receiving ochratoxin A contaminated feed along with different levels of adsorbent showed increase in body weight and normal food conversion ratio (FCR). Reduction in weight gain of chicks in group $B$ may be due to less attraction toward feed. Hypoproteinaemia and low level of somatotropic hormones, result from hepatotoxicity which leads to low feed intake and reduced weight gain in chickens exposed to the experimental ochratoxicosis (Elaroussi et al., 2006). These results are also in line with the 


\section{In Vivo Detoxification of Ochratoxin A by Highly Porous Magnetic Nanocomposites Prepared from Coconut Shell}

Table 9s - Relative body weights ( $\mathrm{g}$ ) of broiler chickens kept on different concentrations of the prepared adsorbent derived from coconut shell.

\begin{tabular}{|c|c|c|c|c|c|c|}
\hline \multirow{3}{*}{$\begin{array}{l}\text { Time period } \\
\text { Week (days) }\end{array}$} & \multicolumn{6}{|c|}{ Groups } \\
\hline & \multicolumn{6}{|c|}{ Ochratoxin A $400 \mu \mathrm{g} / \mathrm{kg}$ feed } \\
\hline & $\mathrm{A}$ & B & $\mathrm{C}$ & $\mathrm{D}$ & $\mathrm{E}$ & $\mathrm{F}$ \\
\hline $1(07)$ & $140.49^{b} \pm 0.6925$ & $140.85^{b} \pm 0.5691$ & $139.93^{b} \pm 0.7110$ & $140.57^{b} \pm 0.5577$ & $141.22^{b} \pm 0.6352$ & $142.57^{\mathrm{a}} \pm 0.6240$ \\
\hline $2(14)$ & $475.78^{a} \pm 3.6336$ & $360.07^{e} \pm 2.4622$ & $396.28^{d} \pm 3.6530$ & $443.89^{c} \pm 3.7919$ & $460.80^{b} \pm 3.9359$ & $471.55^{\mathrm{a}} \pm 3.8013$ \\
\hline $3(21)$ & $920.66^{a} \pm 11.621$ & $579.85^{c} \pm 12.822$ & $626.70^{b} \pm 11.269$ & $913.18^{a} \pm 13.015$ & $931.62^{\mathrm{a}} \pm 12.117$ & $906.25^{\mathrm{a}} \pm 12.019$ \\
\hline $4(28)$ & $1448.2^{\mathrm{a}} \pm 16.681$ & $939.93^{c} \pm 9.7359$ & $1060.4^{b} \pm 7.7620$ & $1445.0^{\mathrm{a}} \pm 17.657$ & $1461.8^{\mathrm{a}} \pm 17.684$ & $1439.7^{\mathrm{a}} \pm 16.866$ \\
\hline $5(35)$ & $1854.0^{\mathrm{ab}} \pm 13.116$ & $1269.4^{d} \pm 12.282$ & $1382.3^{c} \pm 15.297$ & $1823.9^{\mathrm{ab}} \pm 16.098$ & $1863.4^{a} \pm 11.899$ & $1835.0^{\mathrm{ab}} \pm 10.917$ \\
\hline $6(42)$ & $2092.6^{\mathrm{a}} \pm 20.390$ & $1360.9^{c} \pm 15.697$ & $1475.2^{b} \pm 11.534$ & $2065.2^{\mathrm{a}} \pm 30.468$ & $2093.1^{\mathrm{a}} \pm 21.989$ & $2117.6^{a} \pm 20.586$ \\
\hline
\end{tabular}

present study suggesting that reduced weight gain and low feed ingestion was due to hampered protein synthesis by OTA which acts as competitive inhibitor of phenylalanine, preventing the attachment of this specific aminoacid to "Phenylalanine transfer RNA synthetase" enzyme (Kubina et al., 1990).

\section{Relative kidney weight}

Relative kidney weights of broiler chicks ( $1^{\text {st }}$ week to the $6^{\text {th }}$ week) given different levels of adsorbent have been shown in Table (10s). Chicks in group B showed significantly higher values than group $A$ throughout the experimental period. No significant difference was found in the values of kidneys of broiler chicks in group $A, D, E$ and $F$ during the $1^{\text {st }}$ week. No significant difference was observed in the values of relative weight of kidneys in group $A, E$ and $F$ chicks at the end of the $2^{\text {nd }}, 3^{\text {rd }}$ and $6^{\text {th }}$ week. Higher significant difference was observed in the values in group $B$ when compared with the values in group $D, E$ and $F$ fed with different level of the prepared adsorbent. In the present study chicks in group B fed on OTA contaminated feed only (positive control) exhibited increased weight of kidneys. Similar alterations in kidneys have been reported in different avian species (Khadem et al., 2012). The increase in weight of kidney and liver might be associated with the route of elimination of OTA. Toxic effects of OTA upon these organs might have impaired their efficiency of eliminating toxin and resulted in accumulation in these organs.

Table 10s - Relative kidney weights $(\mathrm{g})$ of broiler chickens kept on different concentrations of the prepared adsorbent derived from coconut shell.

\begin{tabular}{|c|c|c|c|c|c|c|}
\hline \multirow{2}{*}{ Time period } & \multicolumn{6}{|c|}{ Groups } \\
\hline & \multicolumn{6}{|c|}{ Ochratoxin A $400 \mu \mathrm{g} / \mathrm{kg}$ feed } \\
\hline Week (days) & A & B & $\mathrm{C}$ & $\mathrm{D}$ & $\mathrm{E}$ & $\mathrm{F}$ \\
\hline $1(07)$ & $0.6567^{b} \pm 0.0176$ & $0.6933^{a} \pm 0.0167$ & $0.6633^{b} \pm 0.0279$ & $0.6567^{b} \pm 0.0120$ & $0.6467^{b} \pm 0.0267$ & $0.6400^{b} \pm 0.0153$ \\
\hline $2(14)$ & $0.7233^{b} \pm 0.0176$ & $0.7833^{a} \pm 0.0176$ & $0.7600^{\mathrm{ab}} \pm 0.0208$ & $0.7467^{\mathrm{ab}} \pm 0.0186$ & $0.7233^{b} \pm 0.0120$ & $0.7067^{b} \pm 0.0260$ \\
\hline $3(21)$ & $0.6533^{c} \pm 0.0233$ & $0.8600^{\mathrm{a}} \pm 0.0252$ & $0.7933^{b} \pm 0.0120$ & $0.6967^{c} \pm 0.0318$ & $0.6767^{c} \pm 0.0120$ & $0.6900^{c} \pm 0.0551$ \\
\hline $4(28)$ & $0.7200^{d} \pm 0.0208$ & $0.9133^{\mathrm{a}} \pm 0.0185$ & $0.8533^{b} \pm 0.0145$ & $0.7900^{c} \pm 0.0115$ & $0.7433^{\mathrm{cd}} \pm 0.0416$ & $0.7333^{d} \pm 0.0219$ \\
\hline $5(35)$ & $0.7333^{\mathrm{d}} \pm 0.0186$ & $0.9900^{\mathrm{a}} \pm 0.0208$ & $0.8633^{b} \pm 0.0328$ & $0.8100^{b c} \pm 0.0115$ & $0.7700^{c d} \pm 0.0115$ & $0.7733^{\mathrm{cd}} \pm 0.0176$ \\
\hline $6(42)$ & $0.7167^{d} \pm 0.0203$ & $1.0267^{\mathrm{a}} \pm 0.0338$ & $0.9333^{b} \pm 0.0145$ & $0.8400^{c} \pm 0.0200$ & $0.7400^{d} \pm 0.0208$ & $0.7500^{d} \pm 0.0200$ \\
\hline
\end{tabular}

\section{Relative liver weight}

Table (11s) has shown the relative liver weights of broiler chicks given different levels of adsorbent (1 $1^{\text {st }}$ week to the $6^{\text {th }}$ week). The mean value of liver weight of broiler chicks in group B was found significantly higher than group $A$ throughout the experimental period. No significant difference was found in the values of the liver of broiler chicks in group $A, D, E$ and $F$ at the end of the forth week. Relative weights of the liver of broiler chicks were not significantly different in groups A, E and F when observed at the end of the $2^{\text {nd }}, 4^{\text {th }}$ and $5^{\text {th }}$ week. Higher significant difference was observed in the values in group $B$ when compared with the values in group D, E and F fed with different level of the prepared adsorbent.

In the present study increase in relative weight of liver in chicks in group B may be due to deposition of fats in the liver formerly described by several researchers (Khadem et al., 2012). No pathological signs were observed in chickens in groups $D, E$ and $F$ fed on ochratoxin A contaminated feed supplemented with different levels of adsorbent and the toxic effects of ochratoxin A were diminished in poultry birds. The main reason behind that is that the adsorbent has showed strong adsorption capacity for ochratoxin A that occurred from the gastrointestinal tract of broilers and thus these chicks were safe from the detrimental 


\section{In Vivo Detoxification of Ochratoxin A by Highly Porous Magnetic Nanocomposites Prepared from Coconut Shell}

Table 11s - Relative weight (g) of the liver of broiler chickens kept on different concentrations of the prepared adsorbent derived coconut shell.

\begin{tabular}{|c|c|c|c|c|c|c|}
\hline \multirow{2}{*}{ Time period } & \multicolumn{6}{|c|}{ Groups } \\
\hline & \multicolumn{6}{|c|}{ Ochratoxin A 400 $\mu \mathrm{g} / \mathrm{kg}$ feed } \\
\hline Week (days) & A & B & $\mathrm{C}$ & $\mathrm{D}$ & $\mathrm{E}$ & $\mathrm{F}$ \\
\hline $1(07)$ & $3.7567^{c} \pm 0.0437$ & $4.0767^{a} \pm 0.0448$ & $3.9567^{\mathrm{ab}} \pm 0.0788$ & $3.8367^{b c} \pm 0.0644$ & $3.7700^{c} \pm 0.0819$ & $3.7967^{b c} \pm 0.0719$ \\
\hline $2(14)$ & $3.9767^{c} \pm 0.0751$ & $4.5033^{\mathrm{a}} \pm 0.0837$ & $4.2433^{\mathrm{ab}} \pm 0.1040$ & $4.1100^{b c} \pm 0.0529$ & $3.9533^{c} \pm 0.1267$ & $3.8800^{c} \pm 0.0361$ \\
\hline $3(21)$ & $4.0567^{b c} \pm 0.0769$ & $4.3867^{\mathrm{ab}} \pm 0.1450$ & $4.4567^{\mathrm{a}} \pm 0.1963$ & $4.1100^{\mathrm{abc}} \pm 0.0529$ & $3.9933^{c} \pm 0.0696$ & $3.8833^{c} \pm 0.054$ \\
\hline $4(28)$ & $4.0500^{\mathrm{b}} \pm 0.0346$ & $5.3000^{\mathrm{a}} \pm 0.2237$ & $4.9467^{\mathrm{a}} \pm 0.0857$ & $4.3267^{b} \pm 0.1539$ & $4.0033^{b} \pm 0.0590$ & $4.0600^{b} \pm 0.0635$ \\
\hline $5(35)$ & $3.8667^{d} \pm 0.0775$ & $6.4367^{\mathrm{a}} \pm 0.2314$ & $5.5167^{b} \pm 0.2367$ & $4.6400^{c} \pm 0.1415$ & $3.8067^{d} \pm 0.0617$ & $3.8867^{d} \pm 0.1126$ \\
\hline $6(42)$ & $3.8833^{\mathrm{a}} \pm 0.0706$ & $6.9233^{b} \pm 0.0996$ & $6.2533^{c} \pm 0.2073$ & $4.9900^{d} \pm 0.0917$ & $3.8633^{d} \pm 0.0353$ & $3.8700^{d} \pm 0.0458$ \\
\hline
\end{tabular}

effects of the mycotoxin (Pasha et al., 2007; Singh et al., 1990).

\section{Relative spleen weight}

Relative spleen weights of broiler chickens given different level of adsorbent have been shown in Table (12s). The mean value of spleen weight of broiler chicks in group B was found significantly higher than group A throughout the experimental period. No significant difference was found in the values of spleen of broiler chicks in group $A, C, D, E$ and $F$ at the end of the $2^{\text {nd }}$ and $3^{\text {rd }}$ week. Higher significant difference was observed in the values in group $B$ when compared with the values in group $C, D, E$ and $F$ fed with different levels of the prepared adsorbents. No alteration was found in relative weight of spleen in chicks in group $E$ and $F$ throughout the whole experimental duration because these groups were provided with adsorbent at concentration of $0.9 \%$ and $1.2 \%(\mathrm{w} / \mathrm{W})$ respectively which indicated maximum effectiveness of the prepared adsorbents. These results were in accordance with the work of other authors, in which they successfully used adsorbents to ameliorate the toxic effects of ochratoxin $A$ in different organs of chicks including spleen and Bursa of fabricius (Singh et al., 1990; Campbell et al., 1983; Pasha et al., 2007; Khadem et al., 2012).

Table 12s - Relative weight ( $\mathrm{g}$ ) of spleen of broiler chickens kept on different concentrations of the prepared adsorbent derived from coconut shell.

\begin{tabular}{|c|c|c|c|c|c|c|}
\hline \multirow{3}{*}{$\begin{array}{l}\text { Time period } \\
\text { Week (days) }\end{array}$} & \multicolumn{6}{|c|}{ Groups } \\
\hline & \multicolumn{6}{|c|}{ Ochratoxin A 400 $\mu \mathrm{g} / \mathrm{kg}$ feed } \\
\hline & A & B & $C$ & $\mathrm{D}$ & $E$ & $\mathrm{~F}$ \\
\hline $1(07)$ & $0.0567^{c} \pm 0.0002$ & $0.1233^{\mathrm{a}} \pm 0.0004$ & $0.0833^{b} \pm 0.0001$ & $0.0700^{b c} \pm 0.0001$ & $0.0633^{c} \pm 0.0002$ & $0.0667^{b c} \pm 0.0002$ \\
\hline $2(14)$ & $0.0567^{c} \pm 0.0002$ & $0.1367^{\mathrm{a}} \pm 0.0145$ & $0.1067^{b} \pm 0.0120$ & $0.0767^{c} \pm 0.0002$ & $0.0733^{c} \pm 0.0003$ & $0.0533^{c} \pm 0.0003$ \\
\hline $3(21)$ & $0.0667^{c} \pm 0.0003$ & $0.1133^{\mathrm{a}} \pm 0.0003$ & $0.0933^{b} \pm 0.0002$ & $0.0767^{c} \pm 0.0004$ & $0.0700^{c} \pm 0.0000$ & $0.0700^{c} \pm 0.0003$ \\
\hline $4(28)$ & $0.0767^{c} \pm 0.0002$ & $0.1500^{\mathrm{a}} \pm 0.0115$ & $0.1133^{b} \pm 0.0003$ & $0.0933^{b c} \pm 0.0003$ & $0.0733^{c} \pm 0.0002$ & $0.0800^{`} \pm 0.0000$ \\
\hline $5(35)$ & $0.0533^{c} \pm 0.0003$ & $0.1067^{a} \pm 0.0145$ & $0.0833^{\mathrm{ab}} \pm 0.0002$ & $0.0767^{b c} \pm 0.0003$ & $0.0633^{b c} \pm 0.0004$ & $0.0733^{b c} \pm 0.0003$ \\
\hline $6(42)$ & $0.0633^{c} \pm 0.0003$ & $0.1167^{\mathrm{a}} \pm 0.0120$ & $0.0967^{\mathrm{ab}} \pm 0.0004$ & $0.0833^{b c} \pm 0.0003$ & $0.0700^{c} \pm 0.0000$ & $0.0667^{c} \pm 0.0003$ \\
\hline
\end{tabular}

\section{Relative weight of bursa of fabricius}

Relative weights of bursa of fabricius of broiler chickens ( $1^{\text {st }}$ week to the $6^{\text {th }}$ week) given different levels of adsorbent have been shown in Table (13s). The mean values of weight of bursa of fabricius in group B were found significantly lower than group A throughout the experimental period. No significant difference was found in the values of bursa of fabricius of broiler chicks in group $A, E$ and $F$ at the end of the $1^{\text {st }}$ and $4^{\text {th }}$ weeks. Higher significant difference was observed in the values in group B when compared with

Table 13s - Relative weight ( $\mathrm{g}$ ) of bursa of fabricius of broiler chickens kept on different concentrations of the prepared adsorbent derived from coconut shell.

\begin{tabular}{lcccccc}
\hline \multirow{2}{*}{ Time period } & \multicolumn{5}{c}{ Groups } \\
\cline { 2 - 7 } & $\mathrm{A}$ & $\mathrm{B}$ & $\mathrm{C}$ & $\mathrm{C}$ & $\mathrm{C}$ & $\mathrm{E}$ \\
\hline Week (days) & $0.1533^{\mathrm{a}} \pm 0.0125$ & $0.1000^{\mathrm{b}} \pm 0.0120$ & $0.1300^{\mathrm{ab}} \pm 0.0115$ & $0.1367^{\mathrm{ab}} \pm 0.0145$ & $0.1500^{\mathrm{a}} \pm 0.0153$ & $0.1567^{\mathrm{a}} \pm 0.0145$ \\
\hline $1(07)$ & $0.1300^{\mathrm{a}} \pm 0.0115$ & $0.0833^{\mathrm{c}} \pm 0.0003$ & $0.1067^{\mathrm{bc}} \pm 0.0120$ & $0.1133^{\mathrm{abc}} \pm 0.0004$ & $0.1433^{\mathrm{ab}} \pm 0.0120$ & $0.1367^{\mathrm{ab}} \pm 0.0145$ \\
$2(14)$ & $0.1100^{\mathrm{abc}} \pm 0.0115$ & $0.0767^{\mathrm{d}} \pm 0.0004$ & $0.0900^{\mathrm{cd}} \pm 0.0003$ & $0.1033^{\mathrm{bc}} \pm 0.0002$ & $0.1267^{\mathrm{a}} \pm 0.0004$ & $0.1133^{\mathrm{ab}} \pm 0.0003$ \\
$3(21)$ & $0.1600^{\mathrm{a}} \pm 0.0153$ & $0.1100^{\mathrm{b}} \pm 0.0115$ & $0.1133^{\mathrm{b}} \pm 0.0003$ & $0.1267^{\mathrm{ab}} \pm 0.0145$ & $0.1633^{\mathrm{a}} \pm 0.0120$ & $0.1633^{\mathrm{a}} \pm 0.0167$ \\
$4(28)$ & $0.1667^{\mathrm{a}} \pm 0.0003$ & $0.1200^{\mathrm{c}} \pm 0.0004$ & $0.1367^{\mathrm{bc}} \pm 0.0002$ & $0.1467^{\mathrm{ab}} \pm 0.0003$ & $0.1500^{\mathrm{ab}} \pm 0.0100$ & $0.1667^{\mathrm{a}} \pm 0.0002$ \\
$5(35)$ & $0.0933^{\mathrm{bc}} \pm 0.0003$ & $0.0633^{\mathrm{d}} \pm 0.0003$ & $0.0800^{\mathrm{c}} \pm 0.0000$ & $0.0867^{\mathrm{c}} \pm 0.0002$ & $0.1067^{\mathrm{ab}} \pm 0.0004$ & $0.1167^{\mathrm{a}} \pm 0.0003$ \\
\hline$(42)$ & & & & &
\end{tabular}




\section{In Vivo Detoxification of Ochratoxin A by Highly Porous Magnetic Nanocomposites Prepared from Coconut Shell}

higher than $D, E$ and $F$ throughout the experimental period. Relative ALT and ALP concentration of broiler chicks was noted non-significant in groups $A, E$ and $F$ at the end of the $3^{\text {rd }}, 4^{\text {th }}, 5^{\text {th }}$ and $6^{\text {th }}$ weeks of the experiment, however lower values were recorded in group $E$ and $F$. Higher significant difference was observed in the values in group $B$ when compared with the values in group $C, D, E$ and $F$ provided with diet supplemented by $400 \mathrm{ppb}$ of OTA along with $0.3 \%, 0.6 \%, 0.9 \%$ and $1.2 \%(\mathrm{w} / \mathrm{w})$ of the prepared adsorbents respectively.

Severe toxicological effects were reported in the serum biochemical parameters of the chicks fed OTA contaminated feed (Verma et al., 2003). In the present study positive control group (fed with ochratoxin contaminated feed only) has shown the highest level of serum of ALT and ALP concentration. However negative control group and adsorbent treated groups have shown comparatively low concentrations. Our results showed that the ALT and ALP levels of chicks in group $A, E$ and $F$ were not significantly different from each other. This indicated that chicks in group $\mathrm{E}$ and $\mathrm{F}$ have normal ALT and ALP concentrations as compared to negative control group (A). Thus our findings revealed that $0.9 \%$ of the prepared nanocomposites (adsorbent) are enough to subside the toxic effects of ochratoxin A in Gallus gallusdomesticus. Such outcomes have also been reported by several researchers (Zahoor \& Khan 2014; Koynarski et al., 2007).

Table 14s - Serum ALT concentration in broiler chickens kept on different levels of the prepared adsorbent derived from coconut shell.

\begin{tabular}{lcccccc}
\hline \multirow{2}{*}{ Time Period } & & \multicolumn{4}{c}{ Group } \\
\cline { 3 - 7 } & $\mathrm{A}$ & $\mathrm{B}$ & $\mathrm{C}$ & $\mathrm{C}$ D & $\mathrm{E}$ & $\mathrm{E}$ \\
\hline Week (days) & $10.750^{\mathrm{b}} \pm 0.2343$ & $11.717^{\mathrm{a}} \pm 0.1683$ & $11.180^{\mathrm{b}} \pm 0.0702$ & $11.113^{\mathrm{b}} \pm 0.1184$ & $11.093^{\mathrm{b}} \pm 0.0644$ & $10.877^{\mathrm{b}} \pm 0.1880$ \\
\hline $1(07)$ & $12.287^{\mathrm{d}} \pm 0.0895$ & $14.680^{\mathrm{a}} \pm 0.1966$ & $13.947^{\mathrm{b}} \pm 0.3671$ & $12.887^{\mathrm{c}} \pm 0.075$ & $12.51^{\mathrm{cd}} \pm 0.067$ & $12.35^{\mathrm{cd}} \pm 0.177$ \\
$2(14)$ & $14.387^{\mathrm{c}} \pm 0.1707$ & $19.687^{\mathrm{a}} \pm 0.461$ & $17.360^{\mathrm{b}} \pm 0.424$ & $15.097^{\mathrm{c}} \pm 0.1122$ & $14.493^{\mathrm{c}} \pm 0.1419$ & $14.393^{\mathrm{c}} \pm 0.167$ \\
$3(21)$ & $12.453^{\mathrm{d}} \pm 0.219$ & $21.353^{\mathrm{a}} \pm 0.469$ & $17.93^{\mathrm{b}} \pm 0.6533$ & $15.513^{\mathrm{c}} \pm 0.18$ & $13.127 \mathrm{~d} \pm 0.0694$ & $12.94^{\mathrm{d}} \pm 0.071$ \\
$4(28)$ & $13.550^{\mathrm{d}} \pm 0.189$ & $36.213^{\mathrm{a}} \pm 0.619$ & $21.307^{\mathrm{b}} \pm 0.482$ & $16.537^{\mathrm{c}} \pm 0.403$ & $13.94^{\mathrm{d}} \pm 0.221$ & $13.887^{\mathrm{d}} \pm 0.233$ \\
$5(35)$ & $12.530^{\mathrm{d}} \pm 0.2163$ & $48.857^{\mathrm{a}} \pm 0.479$ & $25.237^{\mathrm{b}} \pm 0.466$ & $17.567^{\mathrm{c}} \pm 0.257$ & $13.387^{\mathrm{d}} \pm 0.0437$ & $13.277^{\mathrm{d}} \pm 0.0788$ \\
\hline $6(42)$ & & & & &
\end{tabular}

Table 15s - Serum alkaline phosphatase (ALP) concentrations (U/L) in broiler chickens kept on different levels of the prepared adsorbent derived from coconut shell.

\begin{tabular}{|c|c|c|c|c|c|c|}
\hline \multirow{3}{*}{$\begin{array}{l}\text { Time Period } \\
\text { Week (days) }\end{array}$} & \multicolumn{6}{|c|}{ Group } \\
\hline & \multicolumn{6}{|c|}{ Ochratoxin A $400 \mu \mathrm{g} / \mathrm{kg}$ feed } \\
\hline & & B & C & $D$ & $E$ & $\mathrm{~F}$ \\
\hline $1(07)$ & $627.59^{b} \pm 8.171$ & $650.33^{a} \pm 2.905$ & $639.33^{\mathrm{ab}} \pm 1.201$ & $636.00^{\mathrm{ab}} \pm 2.645$ & $629.00^{b} \pm 4.163$ & $628.33^{b} \pm 6.765$ \\
\hline $2(14)$ & $646.50^{b} \pm 2.762$ & $670.00^{a} \pm 1.15$ & $655.33^{b} \pm 5.174$ & $648.33^{b} \pm 4.409$ & $647.67^{b} \pm 3.38$ & $646.67^{b} \pm 4.841$ \\
\hline $3(21)$ & $595.33^{d} \pm 2.8954$ & $690.67^{a} \pm 3.480$ & $671.00^{b} \pm 6.658$ & $630.33^{c} \pm 5.487$ & $599.00^{d} \pm 3.055$ & $597.33^{d} \pm 2.33$ \\
\hline $4(28)$ & $619.07^{d} \pm 4.217$ & $858.33^{a} \pm 10.13$ & $805.00^{b} \pm 5.033$ & $726.33^{c} \pm 6.333$ & $619.33^{d} \pm 1.855$ & $618.67^{d} \pm 0.8819$ \\
\hline $5(35)$ & $665.46^{d} \pm 4.11$ & $961.67^{\mathrm{a}} \pm 4.409$ & $879.00^{b} \pm 0.57$ & $782.00^{c} \pm 7.00$ & $666.33^{d} \pm 3.756$ & $666.33^{d} \pm 2.962$ \\
\hline $6(42)$ & $643.25^{d} \pm 5.01$ & $1124.3^{\mathrm{a}} \pm 8.089$ & $990.67^{b} \pm 5.36$ & $799.00^{c} \pm 6.928$ & $701.33^{d} \pm 6.64$ & $662.33 d \pm 5.044$ \\
\hline
\end{tabular}




\section{In Vivo Detoxification of Ochratoxin A by Highly Porous Magnetic Nanocomposites Prepared from Coconut Shell}

\section{Serum creatinine and urea}

The biochemical analysis of blood serum of broiler chickens for creatinine and urea concentration ( $1^{\text {st }}$ week to the $6^{\text {th }}$ week) is shown in (Table $16 \mathrm{~s}$ and $17 \mathrm{~s}$ ). Mean value of creatinine and urea concentration in group $\mathrm{E}$ and $\mathrm{F}$ were significantly lower than that in group $\mathrm{B}$ and $C$ throughout the experimental period. No significant differences were found in the values of creatinine concentration of broiler chicks in group $A, C, D, E$ and $\mathrm{F}$ at the end of the $1^{\text {st }}$ week of the experiment. Relative creatinine and urea concentrations of broiler chicks were noted non-significant in groups $A, E$ and $F$ at the end of the $3^{\text {rd }}, 4^{\text {th }}, 5^{\text {th }}$ and $6^{\text {th }}$ week of the experiment.

In the present study, creatinine and urea concentration in group B was significantly higher than group A (negative control group) and adsorbent treated groups (particularly D, E and F) at 42 days of experiment. Our findings of increased creatinine and urea values coincide with the results previously reported in White Leghorn and broilers (Kumaret al., 2004). Significantly increased levels of serum creatinine and urea in the OTA treated group in addition to histological changes also confirm impaired renal function and primary site for OTA action. Our results showed that the addition of the adsorbent to ochratoxin A contaminated feed strongly ameliorated the toxic effects of ochratoxin A in Gallus gallusdomesticus. Similar findings have been reported by various researchers. They demonstrated that different adsorbents have the capability to reduce the toxic effect in layer and in chicks when offered ochratoxin A contaminated feed broilers (Kumar et al., 2004).

Table 16s - Serum creatinine concentrations $(\mathrm{mg} / \mathrm{dl})$ in broiler chickens kept on different levels of the prepared adsorbent derived from coconut shell.

\begin{tabular}{lcccccc}
\hline Time Period & \multicolumn{5}{c}{ Group } \\
\cline { 2 - 7 } & $\mathrm{A}$ & $\mathrm{B}$ & $\mathrm{C}$ & $\mathrm{D}$ & $\mathrm{E}$ & \\
\hline Week (days) & $0.4687^{\mathrm{b}} \pm 0.0527$ & $0.6333^{\mathrm{a}} \pm 0.0285$ & $0.5973^{\mathrm{ab}} \pm 0.02$ & $0.4797^{\mathrm{b}} \pm 0.01$ & $0.4723^{\mathrm{b}} \pm 0.0521$ & $0.4613^{\mathrm{b}} \pm 0.0551$ \\
\hline $1(07)$ & $0.4747^{\mathrm{b}} \pm 0.0542$ & $0.7333^{\mathrm{a}} \pm 0.0260$ & $0.7033^{\mathrm{a}} \pm 0.0145$ & $0.5060^{\mathrm{b}} \pm 0.0465$ & $0.4770^{\mathrm{b}} \pm 0.0533$ & $0.4667^{\mathrm{b}} \pm 0.0354$ \\
$2(14)$ & $0.4680^{\mathrm{b}} \pm 0.0367$ & $0.8453^{\mathrm{a}} \pm 0.0230$ & $0.7983^{\mathrm{a}} \pm 0.02$ & $0.4960^{\mathrm{b}} \pm 0.0202$ & $0.4723^{\mathrm{b}} \pm 0.0372$ & $0.4657^{\mathrm{b}} \pm 0.0394$ \\
$3(21)$ & $0.4663^{\mathrm{c}} \pm 0.0388$ & $0.8910^{\mathrm{a}} \pm 0.0260$ & $0.8080^{\mathrm{b}} \pm 0.01$ & $0.4933^{\mathrm{c}} \pm 0.0169$ & $0.4750^{\mathrm{c}} \pm 0.0378$ & $0.4753^{\mathrm{c}} \pm 0.0125$ \\
$4(28)$ & $0.4663^{\mathrm{c}} \pm 0.0388$ & $0.8910^{\mathrm{a}} \pm 0.0260$ & $0.8080^{\mathrm{b}} \pm 0.02$ & $0.4933^{\mathrm{c}} \pm 0.0169$ & $0.4750^{c} \pm 0.0378$ & $0.4753^{\mathrm{c}} \pm 0.0125$ \\
$5(35)$ & $0.4950^{\mathrm{c}} \pm 0.02$ & $1.1900^{\mathrm{a}} \pm 0.1130$ & $0.9963^{\mathrm{b}} \pm 0.01$ & $0.5203^{\mathrm{c}} \pm 0.0170$ & $0.5013 \mathrm{c} \pm 0.01$ & $0.4973^{\mathrm{c}} \pm 0.01$ \\
\hline$(42)$ & & & & & &
\end{tabular}

Table 17s - Serum urea concentrations $(\mathrm{mg} / \mathrm{dl})$ in broiler chickens kept on different levels of the prepared adsorbent derived from coconut shell.

\begin{tabular}{|c|c|c|c|c|c|c|}
\hline \multirow{3}{*}{$\begin{array}{l}\text { Time Period } \\
\text { Week (days) }\end{array}$} & \multicolumn{6}{|c|}{ Group } \\
\hline & \multicolumn{6}{|c|}{ Ochratoxin A 400 $\mu \mathrm{g} / \mathrm{kg}$ feed } \\
\hline & $\mathrm{A}$ & $\mathrm{B}$ & $\mathrm{C}$ & $\mathrm{D}$ & $\mathrm{E}$ & $\mathrm{F}$ \\
\hline $1(07)$ & $11.343^{c} \pm 0.2742$ & $13.153^{\mathrm{a}} \pm 0.0821$ & $12.503^{b} \pm 0.2564$ & $12.093^{b} \pm 0.0521$ & $12.100^{b} \pm 0.0416$ & $11.357^{c} \pm 0.1670$ \\
\hline $2(14)$ & $11.657^{e} \pm 0.1934$ & $15.180^{\mathrm{a}} \pm 0.0361$ & $14.087^{b} \pm 0.0448$ & $13.107 c \pm 0.0581$ & $12.137^{\mathrm{d}} \pm 0.0328$ & $11.663^{\mathrm{e}} \pm 0.1934$ \\
\hline $3(21)$ & $11.660^{d} \pm 0.1858$ & $17.157^{\mathrm{a}} \pm 0.0233$ & $16.180^{b} \pm 0.0907$ & $14.653^{c} \pm 0.1934$ & $12.063^{\mathrm{d}} \pm 0.0437$ & $11.667^{d} \pm 0.1805$ \\
\hline $4(28)$ & $11.727^{c} \pm 0.0982$ & $19.183^{\mathrm{a}} \pm 0.0584$ & $18.810^{\mathrm{a}} \pm 0.1850$ & $15.447^{b} \pm 0.2788$ & $12.143^{c} \pm 0.0517$ & $11.753^{c} \pm 0.1186$ \\
\hline $5(35)$ & $11.750^{\mathrm{e}} \pm 0.2212$ & $21.170^{\mathrm{a}} \pm 0.0917$ & $20.100^{b} \pm 0.1002$ & $16.137^{c} \pm 0.0684$ & $12.160^{d} \pm 0.1069$ & $11.773 d^{e} \pm 0.1129$ \\
\hline $6(42)$ & $11.713^{\mathrm{d}} \pm 0.1053$ & $23.433^{\mathrm{a}} \pm 0.2603$ & $21.207^{b} \pm 0.1087$ & $17.140 \_ \pm 0.1242$ & $12.153^{\mathrm{d}} \pm 0.1444$ & $11.777^{\mathrm{d}} \pm 0.09$ \\
\hline
\end{tabular}

\section{Serum albumen, total proteins and globulin}

Relative serum albumen, total proteins and globulin concentrations of broiler chicks $\left(1^{\text {st }}\right.$ week to the $6^{\text {th }}$ week) given different levels of adsorbent is shown in tables (18s and 19s). No significant difference was found in the values of serum albumen, total proteins and globulin of broiler chicks in groups $A$, $\mathrm{E}$ and $\mathrm{F}$ at the end of the experiment. However the values in group $C$ for the mentioned parameters were significantly different when compared to group $A, E$ and $F$ throughout the experiment. Higher significant difference was observed in the values in group $B$ when compared with the values in groups $D, E$ and $F$ fed with different levels of adsorbent prepared from coconut shell. Chicks in group B (positive control) showed the lowest levels of serum albumen, total proteins and globulin.

In our study low values of serum albumen, total proteins and globulin in chicken fed with ochratoxin A contaminated feed may be due to the declined synthesis in the the liver suffering from hepatic toxicity (Zahoor \& Khan 2015). In serum biochemical parameters, globulin level was significantly lower in chicks in group $B$ (fed with toxin contaminated feed only) than that in group A fed with basal feed (negative control). 


\section{In Vivo Detoxification of Ochratoxin A by Highly Porous Magnetic Nanocomposites Prepared from Coconut Shell}

Table 18s - Serum albumen concentrations $(\mathrm{g} / \mathrm{dl})$ in broiler chickens kept on different levels of the prepared adsorbent derived from coconut shell.

\begin{tabular}{|c|c|c|c|c|c|c|}
\hline \multirow{2}{*}{ Time Period } & \multicolumn{6}{|c|}{ Group } \\
\hline & \multicolumn{6}{|c|}{ Ochratoxin A $400 \mu \mathrm{g} / \mathrm{kg}$ feed } \\
\hline Week (days) & A & B & C & $\mathrm{D}$ & E & $\mathrm{F}$ \\
\hline $1(07)$ & $2.21^{\mathrm{a}} \pm 0.0153$ & $1.96^{d} \pm 0.005$ & $2.0133^{c} \pm 0.008$ & $2.0733^{b} \pm 0.012$ & $2.19^{a} \pm 0.0176$ & $2.20^{a} \pm 0.0289$ \\
\hline $2(14)$ & $2.34^{\mathrm{a}} \pm 0.0145$ & $1.95^{d} \pm 0.0145$ & $2.12^{c} \pm 0.008$ & $2.19^{b} \pm 0.0115$ & $2.36^{\mathrm{a}} \pm 0.0203$ & $2.38^{\mathrm{a}} \pm 0.0208$ \\
\hline $3(21)$ & $2.39^{a} \pm 0.0115$ & $2.05^{d} \pm 0.0115$ & $2.17^{c} \pm 0.0145$ & $2.21^{b} \pm 0.003$ & $2.36^{a} \pm 0.003$ & $2.37^{a} \pm 0.0219$ \\
\hline $4(28)$ & $2.22^{\mathrm{a}} \pm 0.0145$ & $1.95^{d} \pm 0.003$ & $2.04^{c} \pm 0.024$ & $2.10^{b} \pm 0.0120$ & $2.20^{\mathrm{a}} \pm 0.0167$ & $2.21^{\mathrm{a}} \pm 0.0231$ \\
\hline $5(35)$ & $2.40^{\mathrm{a}} \pm 0.0120$ & $2.10^{c} \pm 0.003$ & $2.15^{c} \pm 0.0115$ & $2.2867^{b} \pm 0.003$ & $2.36^{\mathrm{ab}} \pm 0.0557$ & $2.40^{\mathrm{a}} \pm 0.003$ \\
\hline $6(42)$ & $3.56^{a} \pm 0.1255$ & $2.95^{d} \pm 0.0837$ & $3.18^{c} \pm 0.0351$ & $3.32^{b} \pm 0.0536$ & $3.12^{\mathrm{a}} \pm 0.432$ & $3.51^{\mathrm{a}} \pm 0.1273$ \\
\hline
\end{tabular}

Table 19s - Serum total protein concentration $(\mathrm{g} / \mathrm{dl})$ in broiler chickens kept on different levels of the prepared adsorbent derived from coconut shell.

\begin{tabular}{lcccccc}
\hline \multirow{2}{*}{ Time Period } & \multicolumn{5}{c}{ Group } \\
\cline { 2 - 7 } & \multicolumn{5}{c}{ Ochratoxin A 400 $\mu \mathrm{g} / \mathrm{kg}$ feed } \\
\hline Week (days) & $\mathrm{A}$ & $\mathrm{B}$ & $\mathrm{C}$ & $\mathrm{D}$ & $\mathrm{E}$ & $\mathrm{F}$ \\
\hline $1(07)$ & $3.42^{\mathrm{a}} \pm 0.017$ & $3.02^{\mathrm{d}} \pm 0.020$ & $3.06^{\mathrm{d}} \pm 0.027$ & $3.19^{\mathrm{c}} \pm 0.09$ & $3.32^{\mathrm{b}} \pm 0.01$ & $3.39^{\mathrm{ab}} \pm 0.01$ \\
\hline $2(14)$ & $3.55^{\mathrm{a}} \pm 0.01$ & $3.04^{\mathrm{d}} \pm 0.03$ & $3.26^{\mathrm{c}} \pm 0.01$ & $3.37^{\mathrm{b}} \pm 0.021$ & $3.54^{\mathrm{a}} \pm 0.02$ & $3.59^{\mathrm{a}} \pm 0.01$ \\
$3(21)$ & $3.56^{\mathrm{a}} \pm 0.01$ & $2.93^{\mathrm{d}} \pm 0.017$ & $3.19^{\mathrm{c}} \pm 0.25$ & $3.36^{\mathrm{b}} \pm 0.02$ & $3.53^{\mathrm{a}} \pm 0.03$ & $3.56^{\mathrm{a}} \pm 0.02$ \\
$4(28)$ & $3.45^{\mathrm{a}} \pm 0.015$ & $2.68^{\mathrm{c}} \pm 0.017$ & $3.04^{\mathrm{b}} \pm 0.09$ & $3.33^{\mathrm{a}} \pm 0.031$ & $3.42^{\mathrm{a}} \pm 0.01$ & $3.43^{\mathrm{a}} \pm 0.02$ \\
$5(35)$ & $3.56^{\mathrm{a}} \pm 0.02$ & $2.65^{\mathrm{d}} \pm 0.023$ & $3.22^{\mathrm{c}} \pm 0.02$ & $3.40^{\mathrm{b}} \pm 0.01$ & $3.48^{\mathrm{ab}} \pm 0.03$ & $3.57^{\mathrm{a}} \pm 0.04$ \\
$6(42)$ & $3.44^{\mathrm{a}} \pm 0.07$ & $2.46^{\mathrm{d}} \pm 0.03$ & $3.19^{\mathrm{c}} \pm 0.01$ & $3.26^{\mathrm{b}} \pm 0.05$ & $3.42^{\mathrm{a}} \pm 0.07$ & $3.45^{\mathrm{a}} \pm 0.05$ \\
\hline
\end{tabular}

Other authors have also reported the decrease levels of serum albumen, total proteins and globulin in chick fed on ochratoxin contaminated diet only (Khadem et al., 2012). No significant differences were observed among chicks of negative control and those fed on diets containing ochratoxin A plus adsorbent.

The results of broiler chicks in group D, E and $F$ fed on $0.6 \% .0 .9 \%$ and $1.2 \%$ adsorbent respectively showed significant differences as compared to group B fed on ochratoxin A $(400 \mu \mathrm{g} / \mathrm{kg}$ feed) contaminated feed. However no significant difference was noted in the values of chicks in group A (kept on pure feed) and adsorbents treated groups. Similar findings were also presented previously (Kumar et al., 2004). Our results demonstrated that the prepared adsorbents are highly efficient to adsorb and detoxify ochratoxin $A$ in alimentary canal of Gallus gallusdomesticus and passes along feces without any injurious effect.

\section{Clinical signs and behavior}

Scores of the different clinical signs and behavioral studies exhibited by different groups of chicks during each week of the experiment are given in Table (20s). Poultry birds in group A and D, E and F exhibited normal signs and behavior during the experiment. Abnormal signs and behavior were presented by birds in group $B$ as this group exhibit less attraction toward feed and increased water intake throughout the experiment. Also the chicks in this group were depressed. The severity of clinical signs and behavior was at peak at the end of the experimental period. Normal signs and behavior were exhibited by chickens in group $C$ during the first week. The results obtained in the $2^{\text {nd }}$ week were similar to that of the $1^{\text {st }}$ week with slight alterations in behavior, clinical signs and feces consistency. Intensity of clinical signs and behavior was increased with the increase in experimental duration.

Table 20s - Serum globulins concentration $(\mathrm{g} / \mathrm{dl})$ in broiler chickens kept on different levels of the prepared adsorbent derived from coconut shell.

\begin{tabular}{|c|c|c|c|c|c|c|}
\hline \multirow{3}{*}{$\begin{array}{l}\text { Time Period } \\
\text { Week (days) }\end{array}$} & \multicolumn{6}{|c|}{ Group } \\
\hline & \multicolumn{6}{|c|}{ Ochratoxin A 400 $\mu \mathrm{g} / \mathrm{kg}$ feed } \\
\hline & $\mathrm{A}$ & B & $\mathrm{C}$ & $\mathrm{D}$ & $E$ & $\mathrm{~F}$ \\
\hline $1(07)$ & $1.21^{\mathrm{a}} \pm 0.017$ & $1.06^{b} \pm 0.026$ & $1.05^{b} \pm 0.028$ & $1.12^{\mathrm{ab}} \pm 0.0902$ & $1.13^{\mathrm{ab}} \pm 0.042$ & $1.19^{\mathrm{ab}} \pm 0.0441$ \\
\hline $2(14)$ & $1.21^{\mathrm{ab}} \pm 0.041$ & $1.09^{b} \pm 0.0467$ & $1.14^{\mathrm{ab}} \pm 0.047$ & $1.18^{\mathrm{ab}} \pm 0.026$ & $1.18^{\mathrm{ab}} \pm 0.0384$ & $1.21^{\mathrm{a}} \pm 0.0176$ \\
\hline $3(21)$ & $1.17^{a} \pm 0.0173$ & $0.88^{c} \pm 0.0267$ & $1.02^{b} \pm 0.06$ & $1.15^{\mathrm{ab}} \pm 0.0240$ & $1.17^{\mathrm{a}} \pm 0.030$ & $1.19^{a} \pm 0.0319$ \\
\hline $4(28)$ & $1.22^{\mathrm{a}} \pm 0.003$ & $0.73^{c} \pm 0.024$ & $1.0^{\mathrm{b}} \pm 0.047$ & $1.12^{\mathrm{ab}} \pm 0.0203$ & $1.21^{\mathrm{ab}} \pm 0.017$ & $1.22^{\mathrm{a}} \pm 0.003$ \\
\hline $5(35)$ & $1.15^{\mathrm{a}} \pm 0.024$ & $0.55^{c} \pm 0.026$ & $1.07^{b} \pm 0.0208$ & $1.12^{\mathrm{ab}} \pm 0.0203$ & $1.12^{\mathrm{ab}} \pm 0.0291$ & $1.16^{a} \pm 0.033$ \\
\hline $6(42)$ & $1.09^{\mathrm{a}} \pm 0.068$ & $0.43^{b} \pm 0.0406$ & $1.02^{\mathrm{a}} \pm 0.0291$ & $1.03^{\mathrm{a}} \pm 0.042$ & $1.08^{\mathrm{a}} \pm 0.062$ & $1.09^{\mathrm{a}} \pm 0.073$ \\
\hline
\end{tabular}




\section{In Vivo Detoxification of Ochratoxin A by Highly Porous Magnetic Nanocomposites Prepared from Coconut Shell}

Different clinical signs and behavioral alterations in poultry chicks (during ochratoxicosis) have been reported by several researchers (Zahoor \& Khan 2015). They also investigated that ochratoxin toxicity in broilers has resulted in increased water intake and decreased feed consumption; therefore these results are also in accordance to our observed studies. According to our results diarrhea (watery stool), attraction to water intake and depression were some noticeable signs displayed by the broilers fed with

Table 21s - Clinical signs and behavior of broiler chickens kept on different concentration of the prepared adsorbent derived from coconut shell.

\begin{tabular}{|c|c|c|c|c|c|c|c|c|}
\hline \multirow{3}{*}{ Time period } & \multirow{3}{*}{ Clinical signs and behavior } & \multirow{3}{*}{ Range of score } & \multicolumn{6}{|c|}{ Groups } \\
\hline & & & \multicolumn{6}{|c|}{ Ochratoxin A $(400 \mu \mathrm{g} / \mathrm{kg})$ of feed } \\
\hline & & & A & B & $\mathrm{C}$ & $\mathrm{D}$ & $\mathrm{E}$ & $\mathrm{F}$ \\
\hline \multirow{4}{*}{ First week } & Alertness (normal/depressed) & $0-3$ & 0 & 2 & 0 & 0 & 0 & 0 \\
\hline & $\begin{array}{l}\text { Attraction to feed } \\
\text { (normal/less interest) }\end{array}$ & $0-3$ & 0 & 4 & 0 & 0 & 0 & 0 \\
\hline & $\begin{array}{l}\text { Attraction to water } \\
\text { (normal/more/less interest) }\end{array}$ & $0-3$ & 0 & 12 & 0 & 0 & 0 & 0 \\
\hline & $\begin{array}{l}\text { Feces consistency } \\
\text { (normal/ watery) }\end{array}$ & $0-3$ & 0 & 5 & 0 & 0 & 0 & 0 \\
\hline \multicolumn{2}{|c|}{ Cumulative score } & & 0 & 23 & 0 & 0 & 0 & 0 \\
\hline \multirow{4}{*}{ Second week } & Alertness (normal/depressed) & $0-3$ & 0 & 5 & 0 & 0 & 0 & 0 \\
\hline & $\begin{array}{l}\text { Attraction to feed } \\
\text { (normal/less interest) }\end{array}$ & $0-3$ & 0 & 9 & 0 & 0 & 0 & 0 \\
\hline & $\begin{array}{l}\text { Attraction to water } \\
\text { (normal/more/less interest) }\end{array}$ & $0-3$ & 0 & 22 & 1 & 0 & 0 & 0 \\
\hline & $\begin{array}{l}\text { Feces consistency } \\
\text { (normal/ watery) }\end{array}$ & $0-3$ & 0 & 13 & 3 & 0 & 0 & 0 \\
\hline \multicolumn{2}{|c|}{ Cumulative score } & & 0 & 44 & 4 & 0 & 0 & 0 \\
\hline \multirow{4}{*}{ Third week } & Alertness (normal/depressed) & $0-3$ & 0 & 7 & 1 & 0 & 0 & 0 \\
\hline & $\begin{array}{l}\text { Attraction to feed } \\
\text { (normal/less interest) }\end{array}$ & $0-3$ & 0 & 13 & 1 & 0 & 0 & 0 \\
\hline & $\begin{array}{l}\text { Attraction to water } \\
\text { (normal/more/less interest) }\end{array}$ & $0-3$ & 0 & 35 & 2 & 0 & 0 & 0 \\
\hline & $\begin{array}{l}\text { Feces consistency } \\
\text { (normal/ watery) }\end{array}$ & $0-3$ & 0 & 27 & 4 & 0 & 0 & 0 \\
\hline \multicolumn{2}{|c|}{ Cumulative score } & & 0 & 82 & 8 & 0 & 0 & 0 \\
\hline \multirow{4}{*}{ Forth week } & Alertness (normal/depressed) & $0-3$ & 0 & 12 & 1 & 0 & 0 & 0 \\
\hline & $\begin{array}{l}\text { Attraction to feed } \\
\text { (normal/less interest) }\end{array}$ & $0-3$ & 0 & 30 & 2 & 0 & 0 & 0 \\
\hline & $\begin{array}{l}\text { Attraction to water } \\
\text { (normal/more/less interest) }\end{array}$ & $0-3$ & 0 & 52 & 5 & 0 & 0 & 0 \\
\hline & $\begin{array}{l}\text { Feces consistency } \\
\text { (normal/ watery) }\end{array}$ & $0-3$ & 0 & 42 & 7 & 0 & 0 & 0 \\
\hline \multicolumn{2}{|c|}{ Cumulative score } & & 0 & 136 & 15 & 0 & 0 & 0 \\
\hline \multirow{4}{*}{ Fifth week } & Alertness (normal/depressed) & $0-3$ & 0 & 14 & 2 & 0 & 0 & 0 \\
\hline & $\begin{array}{l}\text { Attraction to feed } \\
\text { (normal/less interest) }\end{array}$ & $0-3$ & 0 & 48 & 4 & 0 & 0 & 0 \\
\hline & $\begin{array}{l}\text { Attraction to water } \\
\text { (normal/more/less interest) }\end{array}$ & $0-3$ & 0 & 71 & 7 & 0 & 0 & 0 \\
\hline & $\begin{array}{l}\text { Feces consistency } \\
\text { (normal/ watery) }\end{array}$ & $0-3$ & 0 & 62 & 10 & 0 & 0 & 0 \\
\hline \multicolumn{2}{|c|}{ Cumulative score } & & 0 & 196 & 23 & 0 & 0 & 0 \\
\hline \multirow{4}{*}{ sixth week } & Alertness (normal/depressed) & $0-3$ & 0 & 17 & 3 & 0 & 0 & 0 \\
\hline & $\begin{array}{l}\text { Attraction to feed } \\
\text { (normal/less interest) }\end{array}$ & $0-3$ & 0 & 70 & 6 & 0 & 0 & 0 \\
\hline & $\begin{array}{l}\text { Attraction to water } \\
\text { (normal/more/less interest) }\end{array}$ & $0-3$ & 0 & 86 & 9 & 0 & 0 & 0 \\
\hline & $\begin{array}{l}\text { Feces consistency } \\
\text { (normal/ watery) }\end{array}$ & $0-3$ & 0 & 84 & 10 & 0 & 0 & 0 \\
\hline \multicolumn{2}{|c|}{ Cumulative score } & & 0 & 257 & 28 & 0 & 0 & 0 \\
\hline \multicolumn{2}{|c|}{ Total cumulative score } & & 0 & 738 & 78 & 0 & 0 & 0 \\
\hline
\end{tabular}


ochratoxin A contaminated feed. Such findings have also been observed by Koynarski et al. (2007) in chickens and laying hens fed with different dietary levels of ochratoxin. However adsorbent treated group have shown reduced cumulative score when compared to positive control group (B). Thus the present study clearly indicated the significant effect of prepared adsorbent (derived from coconut shell) in ameliorating the toxic effects of ochratoxin A in broiler chicks.

\section{Relative percentage mortality}

Table (21s) represented relative mortality of chicks, occurred among different groups at the end of each week of the experimental period (for six week). Maximum deaths occurred in B (positive control) that increased with the increase in experimental duration and was found severe at the end of the $6^{\text {th }}$ week of the experiment. However the lowest rate of mortality was observed in the negative control group (fed with ochratoxin free basal feed). Adsorbent treated groups, particularly groups $E$ and $F$ also showed the lowest rate of mortality, like group $A$. The total percentage mortality was as follows: $B>C>D>$ while $A, E, F$ showed same percentage of mortality.

Increase in rate of mortality with increase in ochratoxin exposure duration and dietary OTA concentration (dose) in broilers has been reported by many studies (Elaroussi et al., 2006). In the present study broilers in group B fed with ochratoxin A contaminated feed (without adsorbents) showed the highest percentage of total mortality as compared to adsorbent treated groups $(C, D, E, F)$ and negative control group (A). These outcomes were in agreement with the conclusions of Zahoor et al. (2014). According to them, the highest percentage of mortality was observed in chicken exposed to different dietary levels of mycotoxin contaminated feed. However, least mortality was observed among groups fed with mycotoxin contaminated feed along with different levels of adsorbents.

\section{CONCLUSION}

The comparative evaluation of the mentioned groups has indicated that when ochratoxin A contaminated feed, supplemented with different levels of adsorbent (prepared from coconut shell) was fed to broilers, the ochratoxin A induced signs and symptoms were prevented to great extent in chickens. The reason behind this is that most of the ochratoxin A is adsorbed from the intestinal track of broilers and passed along with feces without any injurious effects. Thus the prepared adsorbent has shown greater in vivo efficacy to ameliorate the toxic effects of OTA in poultry chicks.

\section{ACKNOWLEDGEMENTS}

The authors thank the Higher Education commission of Pakistan for their financial support (Project No: 202515/R\&D/HEC)

\section{REFERENCES}

Arbillaga L, Azqueta A, Van, Delft JH, Lopez de Cerain A. In vitro gene expression data supporting a DNA non-reactive genotoxic mechanism for ochratoxin A. Toxicology and Applied Pharmacology 2007;220:216224.

Arenillas A, Rubiera F, Parra JB, Ania CO, Pis JJ. Surface modification of low cost carbons for their application in the environmental protection. Applied Surface Science 2005;252:619-624.

Campbell ML, May JD, Huff WE, Doerr JA. Evaluation of immunity of young broiler chickens during simultaneous aflatoxicosis and ochratoxicosis. Poultry Science 1983;62:2138-2144.

Elaroussi MA, Mohamed FR, El Barkouky EM, Atta AM, Abdou AM, Hatab MH. Experimental ochratoxicosis in broiler chickens. Avian Pathology2006;35, 263-269.

Gowda NKS, Malathi RU. Suganthi. Effect of some chemical and herbal compounds on growth of Aspergillus parasiticusand aflatoxin production. Animal Feed Science and Technology 2004;116:281-291.

Hadi P, Xu M, Ning C, Ki Lin, CS, McKay G. A critical review on preparation, characterization and utilization of sludge-derived activated carbons for wastewater treatment. Chemical Engineering Journal 2015;260:895906.

Table 22s - Relative percentage mortality of broiler chickens kept on different concentrations of the adsorbent derived from coconut shell.

\begin{tabular}{|c|c|c|c|c|c|c|}
\hline \multirow{3}{*}{$\begin{array}{l}\text { Time Period } \\
\text { Week (days) }\end{array}$} & \multicolumn{6}{|c|}{ Group } \\
\hline & \multicolumn{6}{|c|}{ Ochratoxin A 400 $\mu \mathrm{g} / \mathrm{kg}$ feed } \\
\hline & A & $\mathrm{B}$ & $C$ & $\mathrm{D}$ & $\mathrm{E}$ & $\mathrm{F}$ \\
\hline $1(07)$ & 0 & 0 & 0 & 0 & 0 & 0 \\
\hline $2(14)$ & 1 & 2 & 0 & 1 & 0 & 0 \\
\hline $3(21)$ & 0 & 3 & 1 & 0 & 0 & 1 \\
\hline $4(28)$ & 1 & 7 & 3 & 1 & 2 & 0 \\
\hline $5(35)$ & 1 & 13 & 5 & 3 & 1 & 1 \\
\hline $6(42)$ & 0 & 8 & 3 & 1 & 0 & 1 \\
\hline Total mortality & 3 & 33 & 12 & 6 & 3 & 3 \\
\hline$\%$ mortality & $3 \%$ & $33 \%$ & $12 \%$ & $6 \%$ & $3 \%$ & $3 \%$ \\
\hline
\end{tabular}


Hanif N Q, Naseem M, KhatoonS, Malik N. Prevalence of mycotoxins in poultry rations. Pakistan Journal of Scientific and Industrial Research 2006;49:120-124.

Khadem AA, Sharif SD, Barati M, Borji M. Evaluation of the effectiveness of yeast, zeolite and active charcoal as aflatoxin adsorbent in broiler diets. Global Veterinaria. 2012;8(4):426-432.

Khan FA. Zahoor M. In vivo detoxification of aflatoxin B1 by magnetic carbon nanostructures prepared from bagasse. BMC Veterinary Research 2014;10(1):255.

Koynarski V, Stoev S, Grozeva N, Mirtcheva T, Daskalov H, Mitev J, et al. Experimental coccidiosis provoked by Eimeriaacervulinain chicks simultaneously fed on ochratoxin A contaminated diet. Research in Veterinary Science 2007;82:225-231.

Kubina LF, Harvey RB, Huff WE, Corrier DE, Phillips TD, Rottinghaus GE. Efficacy of hydrated sodium calcium aluminosilicate to reduce the toxicity of aflatoxins and T-2 toxin. Poultry Science 1990;69:1078-1086.

Kuiper-Goodman T, Scott PM. Risk assessment of the mycotoxin ochratoxin A. Biomedical and Environmental Sciences 1989;2(3):179-248.

Kumar A, Jindal N, Shukla CL, Asrani RK, Ledoux, DR, Rottinghaus, GE. Pathological changes in broiler chickens fed ochratoxin $A$ and inoculated with Escherichia coli. Avian Pathology 2004;33:413-417.

Marquize RNM, Harnandez TR. Aflatoxin adsorbent capacity two maxicanaluminosilicates in experimentally contaminated chicks diet. Food Additives \& Contaminants 1995; 12:431-433.

Medina A, Jimenez M, Mateo R, Magan N. Efficacy of natamycin for control of growth and ochratoxin A production by Aspergillus carbonarius strains under different environmental conditions. Journal of Applied Microbiology 2007;103:2234-2239.

Obrien E, Heussner AH, Dietrich DR. Species-Sex-and cell type-specific effects of ochratoxin A and B. Toxicological Sciences 2001;63:256-264.

Ortatatli M, Ogus H, Hatipoglu F, Karaman M. Evaluation of pathological changes in broilers during chronic aflatoxin (50 and 100ppb) and clinoptilolite exposure. Research in VeterinaryScience 2005;78:61-68.

Pasha TN, Farooq MU, Khattak FM, Jabbar MA, Khan AD. Effectiveness of sodium bentonite and two commercial products as aflatoxin adsorbents in diets for broiler chickens. Animal Feed Science and Technology 2007; 132:103-110.

Peckham JC, Doupnik B, Jones $\mathrm{OH}$. Acute toxicity of ochratoxin A and B inchicks. Applied Microbiology 971;21:492-494.

Pfohl-Leszkowicz A, Manderville RA. An update on direct genotoxicity as amolecular mechanism of ochratoxin a carcinogenicity. Chemical Research In Toxicology 2012;25:252-262.

Politis I, Fegeros K, Nitsch S, Schatzmayr G, Kantas D. Use of Trichosporon mycotoxinivorans to suppress the effects of ochratoxicosis on the immune system of broiler chicks. British Poultry Science 2005;46:5865.
Prophet EB. Laboratory methods in histotechnology. $3^{\text {rd }}$ ed. Washington: American Registry of Pathology; 1992. p.279

Raju MV, Devegowda G. Esterified-glucomannan in broiler chicken dietscontaminated with aflatoxin, ochratoxin and T-2 toxin: Evaluation of its binding ability (in vitro) and efficacy as immunomodulator. AsianAustralas. Journal of Animal Science 2002;15:1051-1056.

Sawale GK, Gosh RC, Ravikanth K, Maini S, Rekhe DS. Experimental mycotoxicosis in layer induced by ochratoxin $A$ and its amelioration with herbomineral toxin binder Toxiroak. International Journal of Poultry Science 2009;8:798-803.

Schilter B, Marin-Kuan M, Delatour T, Nestler S, Mantle P, Cavin C. Ochratoxin A: Potential epigenetic mechanisms of toxicity and carcinogenicity. Food Additives \& Contaminants 2005;22:88-93.

Shotwell OL, Hesseltine CW, Goulden ML. Ochratoxin A: occurrence as natural contaminant of a corn sample. Applied Microbiology $1969 ; 17(5): 765-766$

Singh GS, Chauhan VS, Jha Gl, Singh KK.Immunosuppression due to chronic ochratoxicosis in broiler chicken. Journal of Comparative Pathology 1990;103:399-410

Singh NP, McCoy MT, Tice RR, Schneider EL. A simple technique for quantitation of low levels of DNA damage in individual cells. Experimental Cell Research 1988;175:184-191.

Sneha M, Sundaram NM. Preparation and characterization of an iron oxidehydroxyapatite nanocomposite for potential bone cancer therapy. International Journal of nanotechnology 2015;10(Supp 1):99-106.

Stoev SD, Djuvinov D, Mirtcheva T, Pavlov D, Mantle P. Studies on some feed additives giving partial protection against ochratoxin A toxicity in chicks. Toxicology Letters 2002;135:33-50.

Wang GH, Xue CY, Chen F, Ma YL, Zhang X B, Bi YZ, et al. Effects of combinations of ochratoxin $A$ and $\mathrm{T}-2$ toxin on immune function of yellow-feathered broiler chickens. Poultry Science 2009;88:504-510.

Van der Stegen GHD, Essens PJM, Van der Lijn J. Effect of roasting conditions on reduction of ochratoxin $A$ in coffee. Journal of Agricultural and Food Chemistry2001;49: 4713-4715.

Verma JT, Johri BK, Swain S, Ameena S. Effect of graded levels of aflatoxin ochratoxin and their combinations on the performance and immune response of broilers. British Poultry Science 2004;45:512-518.

Zahoor M, Khan FA. Adsorption of aflatoxin B1 by magnetic carbon nanocomposite prepared from bagasse. Arabian Journal of Chemistry 2014;11(5):729-738.

Zahoor M, Khan FA. Aflatoxin B1 detoxification by magnetic carbonnanostructures prepared from maize straw. Desalination and water treatment 2015:57(25):11893-11903. 
